\title{
The Wolf-Rayet stars in M 31
}

\section{Analysis of the late-type WN stars}

\author{
A. Sander, H. Todt, R. Hainich, and W.-R. Hamann
}

\begin{abstract}
Institut für Physik und Astronomie, Universität Potsdam, Karl-Liebknecht-Str. 24/25, 14476 Potsdam, Germany e-mail: [ansander;htodt] @astro.physik.uni-potsdam.de
\end{abstract}

Received 12 December 2013 / Accepted 22 January 2014

\section{ABSTRACT}

\begin{abstract}
Context. Comprehensive studies of Wolf-Rayet stars were performed in the past for the Galactic and the LMC population. The results revealed significant differences, but also unexpected similarities between the WR populations of these different galaxies. Analyzing the WR stars in M31 will extend our understanding of these objects in different galactic environments.

Aims. The present study aims at the late-type WN stars in M31. The stellar and wind parameters will tell about the formation of WR stars in other galaxies with different metallicity and star formation histories. The obtained parameters will provide constraints to the evolution of massive stars in the environment of M 31.

Methods. We used the latest version of the Potsdam Wolf-Rayet model atmosphere code to analyze the stars via fitting optical spectra and photometric data. To account for the relatively low temperatures of the late WN10 and WN11 subtypes, our WN models have been extended into this temperature regime.

Results. Stellar and atmospheric parameters are derived for all known late-type WN stars in M 31 with available spectra. All of these stars still have hydrogen in their outer envelopes, some of them up to $50 \%$ by mass. The stars are located on the cool side of the zero age main sequence in the Hertzsprung-Russell diagram, while their luminosities range from $10^{5}$ to $10^{6} L_{\odot}$. It is remarkable that no star exceeds $10^{6} L_{\odot}$

Conclusions. If formed via single-star evolution, the late-type WN stars in M 31 stem from an initial mass range between 20 and $60 M_{\odot}$. From the very late-type WN9-11 stars, only one star is located in the S Doradus instability strip. We do not find any late-type WN stars with the high luminosities known in the Milky Way.
\end{abstract}

Key words. stars: massive - stars: evolution - stars: mass-loss - stars: Wolf-Rayet - stars: atmospheres - stars: winds, outflows

\section{Introduction}

M31 or the Andromeda galaxy is the largest member of the Local Group, which contains only two other spiral-type galaxies, M33 and the Milky Way. Because of the very low foreground extinction towards M31 and the known distance, this galaxy is ideal for studying bright, resolvable stellar objects, such as Wolf-Rayet (WR) stars. Analyzing the WR stars in M31 will extend our knowledge about the formation and evolution of massive stars in other galaxies and allow for a comparative analysis of their WR populations.

Previous studies chiefly focused on the WR population in the Milky Way and the Magellanic Clouds. One of the reasons for this limitation was the paucity of available spectra of WR stars in other galaxies. The advent of multi-object spectroscopy has greatly facilitated such observations. Good quality spectra of extragalactic WR stars are now becoming available, allowing for qualitative analyses. A large set of WR spectra for M 31 have been published by Neugent et al. (2012) and provided the basis for this work. Photometric data of extragalactic WR stars are contained in the Local Group Galaxy Survey (LGGS) by Massey et al. (2006).

Spectroscopically, the WR stars are divided into the WN, $\mathrm{WC}$, and WO subclass. The WN stars show prominent nitrogen emission lines in their spectra while the WC and WO stars have

\footnotetext{
* Appendices are available in electronic form at http: //www . aanda . org
}

prominent carbon and oxygen emission lines. In contrast to the WC stars, the oxygen emission lines are significantly stronger in the WO star spectra, including a prominent $\mathrm{O}$ VI emission line at 3811-34 $\AA$. All subclasses are further split into a sequence of subtypes, defined by the equivalent width or peak ratio of certain emission lines (Crowther et al. 1995; van der Hucht 2001). For the WN stars, these are the subtypes WN2 to WN11. The WN2 to WN6 subtypes are also called "early" types, while the WN8 to WN11 are referred to as "late" subtypes. The WN7 subtype is something in between, although it is often included under the late subtypes. The WN9 to WN11 subtypes were introduced by Crowther et al. (1995) as a finer replacement of the former Ofpe/WN9 classification and are sometimes referred to as "very late" WN types (WNVL: very late WN).

In contrast to the early subtypes, late-type WN stars typically have significant amounts of hydrogen (Hamann et al. 2006). In the past years, hydrogen-rich WN stars, sometimes classified as WNh, have become an interesting topic of research. Studies such as Gräfener et al. (2011) have pointed out that at least a significant fraction of these stars are not "classical" WR stars in the sense that they are stripped cores of evolved massive stars. Instead, WN stars of this kind are most likely core-hydrogen burning, and they form the most luminous group within the WR population. The most luminous WR stars in the Large Magellanic Cloud (LMC) and the Milky Way are in fact such hydrogen-rich WN stars (Barniske et al. 2008; Crowther et al. 2010). 
In this paper we analyze the late-type WN stars with subtypes ranging from WN7 to WN11. Apart from two stars with insufficient spectra, we cover the whole known sample of latetype WN stars in M 31 with available optical spectra.

This present paper builds on our recent work that analyzes the WR star populations in the Milky Way (Hamann et al. 2006; Sander et al. 2012) and the LMC (Hainich et al. 2014). Extending the analysis to galaxies beyond the Milky Way may in principle allow study of WR stars at higher metallicities. According to Zaritsky et al. (1994), the metallicity in M 31 has a value of $\log (\mathrm{O} / \mathrm{H})+12=8.93$, which is a bit higher than the solar value of 8.7 (Esteban \& Peimbert 1995). However, as in our Milky Way, the metallicity is not uniform across the whole galaxy but instead increases towards the central bulge. Figure 1 shows the positions of the analyzed stars in M 31. It is significant that none of the known late-type WN stars in M31 are located in the inner part of this galaxy. Instead the stars mostly reside in a zone between $9 \mathrm{kpc}$ and $15 \mathrm{kpc}$ from the center of M 31 . It is exactly this region that van den Bergh (1964) found to be the most active in forming stars. Because of their non-central location it might be that the metallicity of our sample is not significantly higher than the one used in our Galactic WN models (cf. Hamann \& Gräfener 2004).

The distance to M31 is well known $(d=0.77 \mathrm{Mpc}$, Karachentsev et al. 2004). This offers a big advantage for analyzing of stellar populations. Among the Galactic stars, there is only a limited subsample for which distances can be inferred from cluster or association membership. Analyzing the M31 WN stars will therefore allow us to crosscheck our findings from the Galactic WR analyses and compare them with the LMC results where we have a different type of galaxy and lower metallicity.

In the next section, we briefly characterize the stellar wind models used in this work. In Sect. 3 we report the results and compile the obtained stellar parameters. In Sect. 5 we discuss the results and compare them with theoretical stellar evolution tracks. The conclusions are drawn in the Sect. 6. In the Appendix A we show all spectral fits obtained in this work.

\section{Stellar wind models}

We employ stellar atmosphere models computed with the the Potsdam Wolf-Rayet (PoWR) code. For the present study we extend the published WN model grids (e.g., Hamann \& Gräfener 2004) to lower temperatures in order to cover the very late subtypes WN10 and WN11. The PoWR models assume a spherically symmetric, stationary outflow. The models cover the whole stellar atmosphere and wind, starting at the inner boundary $R_{*}$, which is defined at a Rosseland optical depth of $\tau=20$. The stellar temperature $T_{*}$ is defined via Stefan-Boltzmann's law as the effective temperature at $R_{*}$.

The velocity field is described as a so-called $\beta$-law in the supersonic part. Here we use a value of $\beta=1$. Wind inhomogeneities are treated in the so-called "microclumping" approximation assuming optical thin clumps, which have a density value increased by a factor of $D$ compared to a smooth wind with same mass-loss rate. This factor is set to $D=4$ in all models, similar to our Galactic WN models used in earlier works, such as Hamann et al. (2006) and Liermann et al. (2010). In principle, the PoWR code can account for inhomogeneities of any optical depth (Oskinova et al. 2007), but we do not consider macroclumping in this work. The interclump space is assumed to be void, and thus the volume-filling factor $f_{V}$ is simply given by $f_{V}=D^{-1}$.

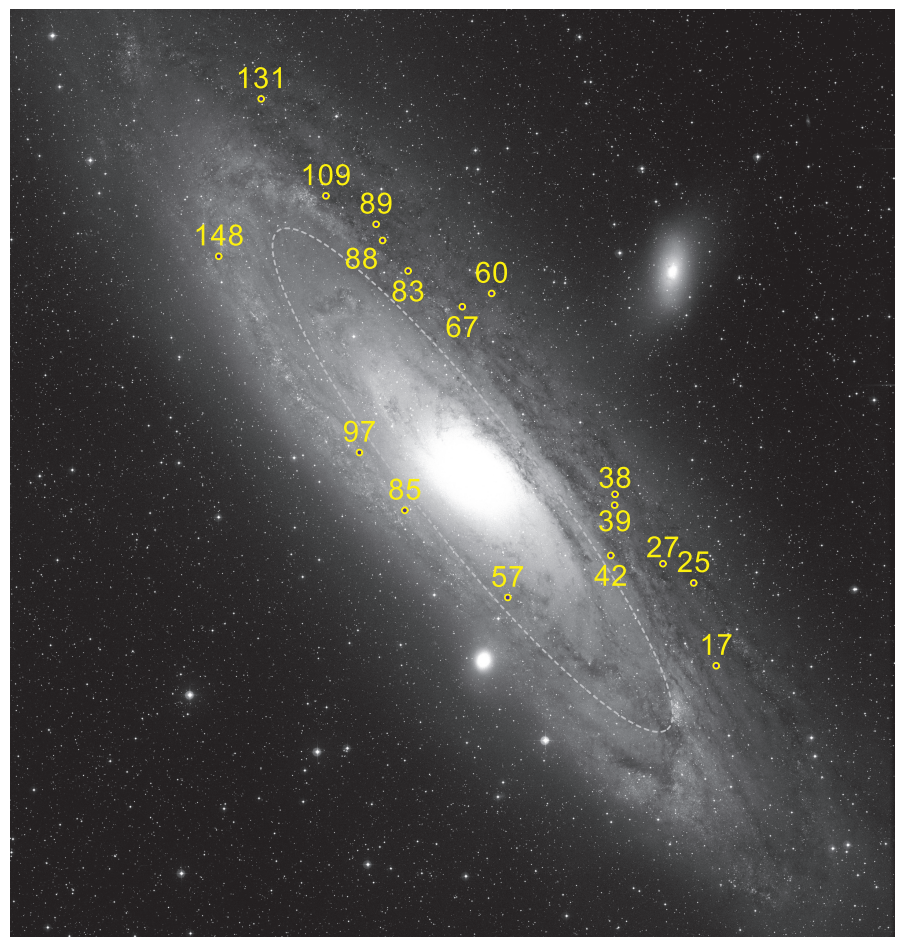

Fig. 1. Positions of the analyzed WN stars in M 31. Numbers correspond to the M31WR numbers as in Table 2. The background image is taken from Palomar STScI DSS. The size of the image is $\approx 1.9^{\circ} \times 2^{\circ}$. The gray dashed ellipse roughly corresponds to a radius of $9 \mathrm{kpc}$ around the center of M31, corresponding to the inner boundary of the region with the most star formation (van den Bergh 1964).

As discussed in in Hamann \& Gräfener (2004), there are two main parameters that describe the overall appearance of a normalized Wolf-Rayet emission line spectrum, namely the stellar temperature $T_{*}$ and the so-called "transformed radius"

$R_{\mathrm{t}}=R_{*}\left[\frac{v_{\infty}}{2500 \mathrm{~km} \mathrm{~s}^{-1}} \mid \frac{\dot{M} \sqrt{D}}{10^{-4} M_{\odot} / \mathrm{yr}}\right]^{\frac{2}{3}}$,

which was introduced by Schmutz et al. (1989) to describe that stars with the same value of $R_{\mathrm{t}}$ have roughly the same spectral appearance. While the bulk of WN models have been calculated with a terminal wind velocity of $v_{\infty}=1000 \mathrm{~km} \mathrm{~s}^{-1}$, we adopt a value of $v_{\infty}=500 \mathrm{~km} \mathrm{~s}^{-1}$ for the latest subtypes (cf. Table 1). In some cases where the lines clearly indicate even lower values, special models are calculated to further improve the matching of the observed spectral lines.

\section{The studied sample}

\subsection{Name convention}

We analyzed the whole sample of WN stars with late subtypes in M31 stars from Neugent et al. (2012). Their spectra were taken with the $6.5 \mathrm{~m}$ telescope at the MMT Observatory in Arizona using the Hectospec multi-object fiber-fed spectrograph. The useful spectral range extends from 3700 to $7500 \AA$. The spectral resolution is $6 \AA$. Instead of using the long LGGS names from Massey et al. (2006), we introduce a WR number system, following Table 5 from Neugent et al. (2012), which has already been sorted by coordinates. Thus J003857.19+403132.2 becomes M31WR 1, J003911.04+403817.5 becomes M31WR 2, and so on. In the text of this work, we further shorten M31WR 1 
Table 1. Fundamental parameters of the WNh model grids.

\begin{tabular}{|c|c|c|c|}
\hline \multirow{2}{*}{$\begin{array}{l}\text { Parameter } \\
X_{\mathrm{H}}\end{array}$} & \multicolumn{3}{|c|}{ WNh grids } \\
\hline & $50 \%$ & $35 \%$ & $20 \%$ \\
\hline$X_{\mathrm{He}}$ & $48 \%$ & $63 \%$ & $78 \%$ \\
\hline$X_{\mathrm{C}}$ & & $0.01 \%$ & \\
\hline$X_{\mathrm{N}}$ & & $1.5 \%$ & \\
\hline$X_{\mathrm{Fe}}{ }^{a}$ & & $0.14 \%$ & \\
\hline$T_{*} / \mathrm{kK}$ & & $18 . .45$ & \\
\hline$R_{\mathrm{t}} / R_{\odot}$ & & $4 . .100$ & \\
\hline $\log L / L_{\odot}$ & & 5.3 & \\
\hline$v_{\infty} / \mathrm{km} \mathrm{s}^{-1}$ & & $500 / 1000^{l}$ & \\
\hline$D$ & & 4 & \\
\hline
\end{tabular}

Notes. ${ }^{(a)}$ Generic element, including the iron group elements $\mathrm{Fe}, \mathrm{Sc}$, Ti, V, Cr, Mn, Co, and Ni. See Gräfener et al. (2002) for relative abundances. ${ }^{(b)}$ Standard models in the whole parameter range were available with 500 and $1000 \mathrm{~km} \mathrm{~s}^{-1}$. For certain stars, additional models with especially adjusted (often lower) values were calculated.

to \#1 to improve the readability. The full LGGS names are given in the figure captions and also in the spectral fits in Appendix A.

This study is focused on WN stars that exhibit hydrogen in their wind. This comprises all WN8 to WN11 subtypes in the sample from Neugent et al. (2012) but also the two WN7 stars \#38, \#57, and \#88. The WN7 star \#86 most likely shows a composite spectrum (see Sect. 3.2) and is therefore excluded from our analysis. For the two other stars classified as WN7, \#1 and \#41, the quality of the spectra is not sufficient for a quantitative analysis. The two objects classified as WN6-7, \#10 and \#21, are apparently hydrogen-free and therefore their analyses is postponed to our forthcoming study of the WNE class.

Our remaining sample thus comprises 17 stars (3 WN7, 1 WN7-8, 6 WN8, 3 WN9, 3 WN10, 1 WN11). Compared to the total number of $92 \mathrm{WN}$ stars in M 31 (Neugent et al. 2012), the number of WNL stars $(\approx 20)$ is surprisingly small. Among the Galactic single WN stars, Hamann et al. (2006) found that 26 out of 59 objects belong to the WNL class, taking detectable hydrogen as criterion. As M31 is bigger than our own Galaxy, one would expect that the total number of WN stars is also higher. We therefore suggest that the known sample of WR stars in M 31 is actually far from being complete, especially for the very late WN9 to WN11 subtypes. A lot of these stars have been discovered in the Milky May in the past decade, when infrared spectroscopy became available and obscured clusters could be studied, e.g., in the Galactic center (Krabbe et al. 1995; Martins et al. 2008; Liermann et al. 2010) or in the G305 star forming complex (Davies et al. 2012). In the current Galactic WR star catalog ${ }^{1}$, there are roughly 60 WN9 stars listed, but only eight of them have optical photometry, and for the rest the classification relies on limited infrared data. One can therefore expect that the current sample of late-type WN stars in M 31 is not complete. It is difficult to quantify how much we are really missing. If the star formation rate (SFR) was roughly the same as in the Milky Way, it would be a large amount. However, if the SFR is significantly lower and comparable to M33, as Neugent et al. (2012) inferred from the integrated $\mathrm{H} \alpha$ luminosity, the number of missing latetype WN stars would propably be only close to our sample size. Neugent et al. (2012) further state that for WN9-11 stars the problem of missing objects is not only due to obscuration, but also to the detection method, which was not sensitive to those subtypes.

\footnotetext{
http://pacrowther.staff.shef.ac.uk/WRcat/
}
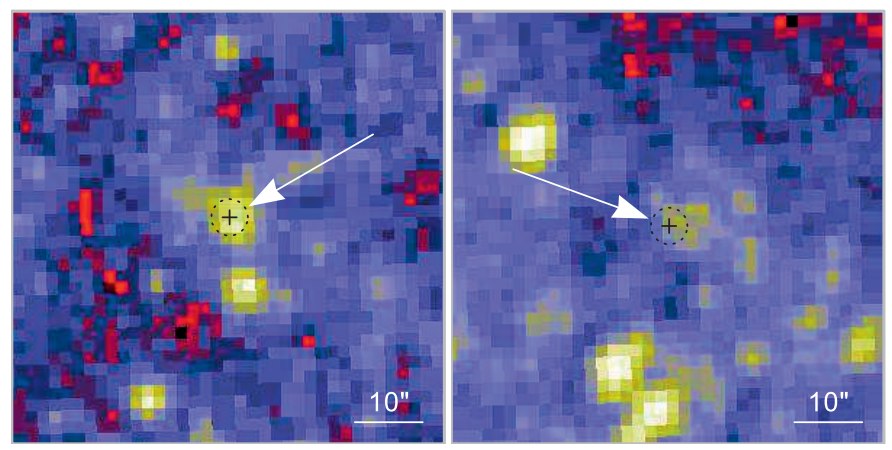

Fig. 2. Surroundings of M31WR 86 (left) and M31WR 109 (right): the black cross marks the location of the object referring to the coordinates in Neugent et al. (2012). The slight offset between the sources in DSS images and Neugent et al. (2012) is seen for all WR stars. The dotted circle corresponds to a distance of $10 \mathrm{pc}$ around the objects. The images were retrieved with SkyView from the Palomar STScI DSS and have a size of $1.06^{\prime} \times 1.06^{\prime}$ each.

\subsection{Binarity}

It is known that a large fraction of the massive stars are actually in binary systems (see, e.g., Vanbeveren \& Conti 1980; Barbá et al. 2010; Sana et al. 2013). The detection of close binarity requires detailed monitoring of photometric or radial-velocity variations. Such studies had not yet been performed for WR stars in M31. At the large distance of M31, even a small cluster may appear as a single star. Therefore one may ask how many of our sample stars are still undetected binaries. In the case of a luminous companion, the spectrum would in fact be composite, and the analysis may lead to wrong stellar parameters.

One indicator of a composite spectrum is the so-called "dilution" of WR emission lines. In a system with a WR star and one or more luminous components without significant emission lines, the continua of all stars will add up, while the emission lines originate in the WR component alone. In the normalized spectrum, the emission lines will therefore be significantly weaker compared to a single WR star of the same subtype. While the dilution criterion seems to be a rather good indicator of WC binaries (Sander et al. 2012), Hamann et al. (2006) argue that it is not sufficient for WN stars because it is possible to find appropriate stellar atmosphere models for WN stars with "weaker" lines.

Even if apparent dilution of emission lines might not be a sufficient binarity criterion, the absence of dilution is a rather good indicator that a star at least does not have a luminous companion. In our sample of late type WN stars, only two objects show the typical signs of diluted emission lines. The WN7 star \#86 shows the typical broad lines with low peak heights, e.g. of He II $4686 \AA$, and is therefore excluded here. The situation is less clear for the WN8 star \#109. The relatively broad N III 4634-42 $\AA$ complex cannot be reproduced by the same model that fits the rest of the lines. The luminosity of $\log L / L_{\odot}=$ 5.9 is slightly higher than for the majority of stars in our sample. Nevertheless, we feel that these indications are not strong enough to exclude \#109 from our sample.

Apart from the spectral appearance, we also took a look at the surroundings of the binary candidates using the Palomar STScI DSS images. While other stars in our sample appear as a single source, \#86 (left panel in Fig. 2) appears to be in a crowded region and might even be a member of a small cluster, so the spectrum is really not from a single Wolf-Rayet star, and we exclude the star from further study. The star \#109 (right panel 
in Fig. 2) lacks the bright appearance of most other sources, but the situation remains unclear so we keep this star in the sample.

\section{Results}

\subsection{Fitting the normalized spectra}

In a first step of our analysis, we identify the best-fitting model for each star from our standard grid in the $T_{*}-R_{\mathrm{t}}$-plane. The focus is usually on the unblended helium lines. In several cases, the helium lines are in good agreement with the observation, but the observed hydrogen lines are stronger than predicted by our models with $X_{\mathrm{H}}=20 \%$. For such cases we calculate two additional grids of models with hydrogen abundances of $35 \%$ and $50 \%$, respectively (see Table 1). Together with our standard WN grid, which uses $X_{\mathrm{H}}=20 \%$, this allows us to estimate the hydrogen fraction.

In a further step, the terminal wind velocity $v_{\infty}$ is adjusted if the line width is significantly over- or underestimated by our standard grid models. Individual models are then calculated until the line width is reproduces satisfactorily.

M31 has a mean radial velocity (relative to the sun) of $v_{\text {rad }}=-301 \mathrm{~km} \mathrm{~s}^{-1}$ (Karachentsev et al. 2004). For our line fits, all observed spectra have to be shifted according to this value. In addition, several stars show a significant individual radial velocity. In such cases, we apply an additional shift until the observation matches with the model. The resulting peculiar radial velocities are included in Table 2. Obtaining precise radial velocities is not an aim of this study, and therefore we accept a relatively large error margin of $\pm 25 \mathrm{~km} \mathrm{~s}^{-1}$. Nevertheless, the obtained results are in good agreement with the known rotation of M31 (e.g., Rubin \& Ford 1970).

Figure 3 shows a typical example of a normalized line fit. The example spectrum demonstrates how narrow the emission lines of these late-type WN stars are. In this case, a terminal velocity of $v_{\infty}=500 \mathrm{~km} \mathrm{~s}^{-1}$ is used, while other stars have even lower velocities (see Table 2).

There are a few lines in the spectra that cannot be reproduced by our models. Apart from a terrestrial absorption feature around $5575 \AA$, there are several interstellar emission lines, such as [O II] $3727 \AA$, [O III] $5007 \AA$, [N II] $6548 \AA$ [N II] $6583 \AA$, and [S II] $6717 / 31 \AA$. These forbidden lines are typical of the so-called DIG (diffuse interstellar gas) that is associated with prominent spiral arms in M 31 (Greenawalt et al. 1997). The two [N II] lines at $6548 \AA$ and $6583 \AA$ can overlap with $\mathrm{H} \alpha$ in earlytype WN spectra. However, for the late types studied here, $\mathrm{H} \alpha$ is narrower and hence well separated from these [N II] lines.

With the help of the three WNh grids we can estimate the hydrogen content of the sample stars to a precision of approximately $\pm 10 \%$ (cf. Table 2 ). All stars of our sample contain a detectable amount of hydrogen, mostly with a mass fraction between $20 \%$ and $35 \%$. For four objects, the WN9 star \#27 and the WN8 stars \#39, \#85, and \#109, a model with 50\% is required for reproducing the observed strength of the Balmer lines.

From the fit of the normalized spectra we obtain the stellar temperature $T_{*}$ and the transformed radius $R_{\mathrm{t}}$ for all stars in our sample (cf. Table 2 and Fig. 4). The location of the stars in the $R_{\mathrm{t}^{-}}$ $T_{*}$-plane reflects their spectral subtypes. There is no correlation between the hydrogen content and subtype or temperature.

The terminal wind velocities $v_{\infty}$ are higher for stars with less hydrogen. The three WN7 stars \#38, \#57, and \#88 require models with $v_{\infty}=1000 \mathrm{~km} \mathrm{~s}^{-1}$ in contrast to the rest ${ }^{2}$ of the analyzed WNh stars.

For two stars, \#42 and \#60, the fitting process caused some trouble. The star \#42 has not been classified in detail so far because of limited available classification lines. Because of the highly uncertain results in this case, this star is excluded from most of the further discussions in this work, and \#60 shows strong $\mathrm{He}$-lines that could not be reproduced. Details for both objects are discussed in Appendix B. The complete set of all spectral fits is located in the following Appendix A.

\subsection{Luminosities}

After the best-fitting model has been selected by means of the line fit, the luminosity of the model now has to be adjusted to reproduce the observed absolute fluxes of the star. The grid models were calculated with a luminosity of $\log L / L_{\odot}=5.3$ and are now scaled such that the observed spectral energy distribution (SED) is matched. Scaling the luminosity does not affect the fit of the normalized line spectrum, as long as the transformed radius $R_{\mathrm{t}}$ is kept.

At the same time, the interstellar reddening color excess $E_{B-V}$ has to be determined, too. The reddening is relatively small for all our objects in our sample. We apply the reddening law by Fitzpatrick (1999). For M 31 we adopt a distance modulus of $\mathrm{DM}=24.4$ corresponding to $d=0.77 \mathrm{Mpc}$ (Karachentsev et al. 2004), and geometrically dilute the model flux accordingly.

We use the UBVRI photometry from Massey et al. (2006) to describe the spectral energy distribution, since the spectra from Neugent et al. (2012) are not flux-calibrated. For a few objects (\#10, \#21, \#25, and \#42) there are UV flux measurements from the XMM Optical/UV Monitor (XMM-OM) available from Page et al. (2012). An example of an SED fit is shown in Fig. 5, the resulting luminosities and $E_{B-V}$ color excesses are compiled in Table 2.

\subsection{Mass-loss rates}

The empirical mass-loss rates are plotted in Fig. 6 against the luminosity. There is no significant correlation for the whole sample, but one notices a certain trend for the stars with only $20 \%$ hydrogen with the exception of the previously discussed \#42 and \#60. There is no subtype sequence visible in the $\dot{M}$ - $L$-diagram; instead, the very late WN10 and WN11 are closer to the WN7 stars than most of the WN8 and WN9 stars. This underlines that using the three WN9 to WN11 subtypes, introduced by Crowther et al. (1995) instead of the former Ofpe/WN9 classification, is indeed helpful as the different subtypes really have different stellar parameters.

When comparing these results with the relations suggested by Nugis \& Lamers (2000), it becomes clear that the mass-loss rates of the WN10 and WN11 stars are higher than expected from their luminosities. Interestingly, Galactic WN stars of very late subtype, such as the "peony star" WR102ka, show the opposite behavior. The highest luminosities in our sample are found among the WN8 and WN9 stars.

In Fig. 7 we plot the so-called modified wind momentum $D_{\text {mom }}=\dot{M} v_{\infty} \sqrt{R_{*}}$ over luminosity. Now we can find a nice relation for all those stars with low $\left(X_{\mathrm{H}} \approx 0.2\right)$ hydrogen content. Such stars are likely to represent the "classical" Wolf-Rayet

\footnotetext{
2 We also give $v_{\infty}=1000 \mathrm{~km} \mathrm{~s}^{-1}$ for \#109, but as discussed in Sect. 3.2, this object is suspicious and might not be a single star.
} 
A. Sander et al.: The Wolf-Rayet stars in M31. I.

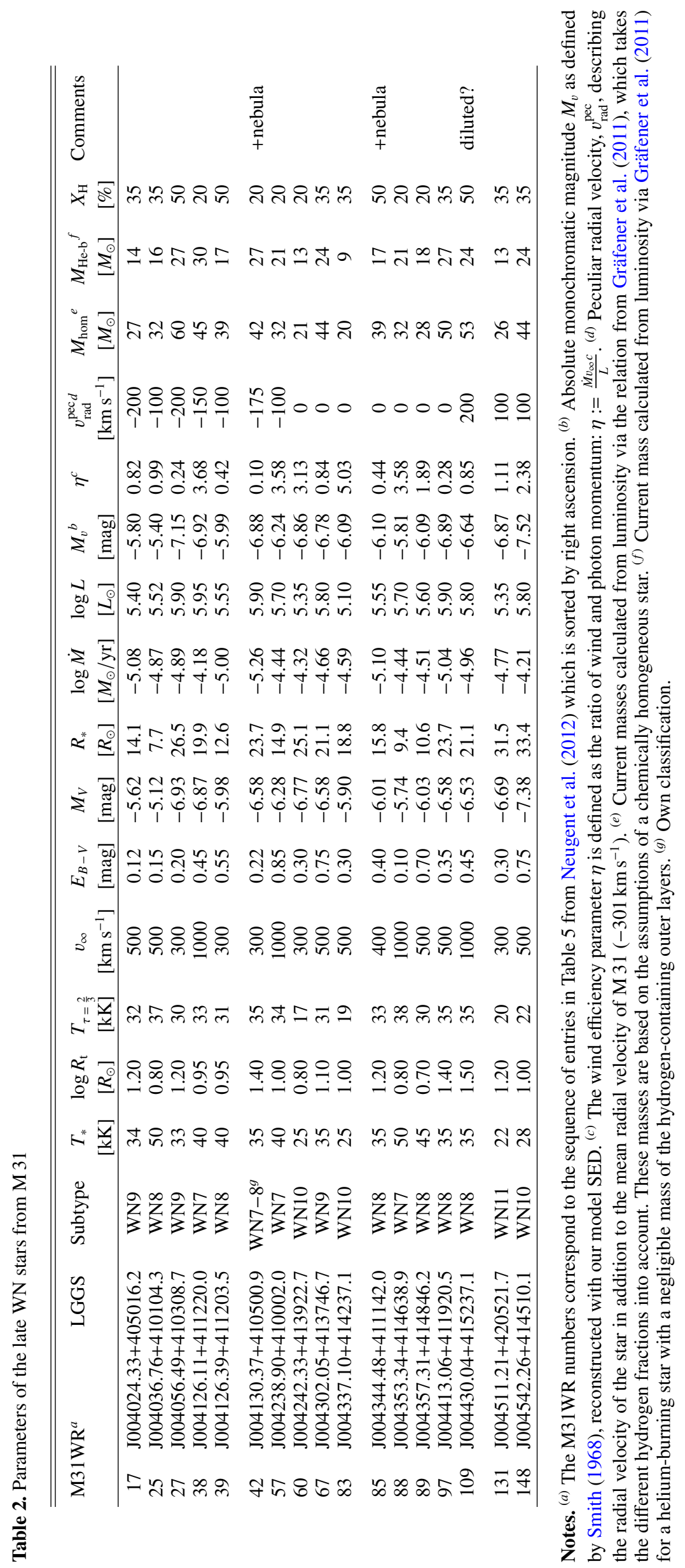




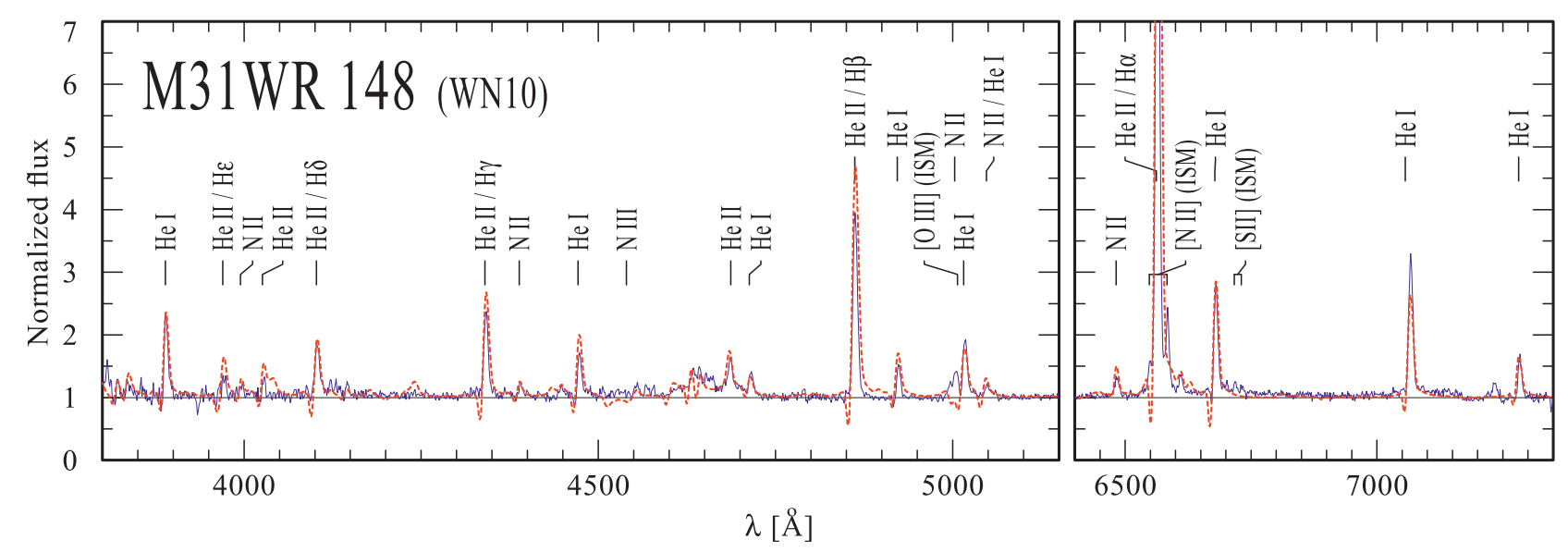

Fig. 3. Optical spectrum of M31WR 148 alias LGGS J004542.26+414510.1. The solid thin blue line shows the observation from Neugent et al. (2012), and the best-fitting PoWR model is overplotted as red thick dotted line. Important lines, including those from interstellar gas, are identified.

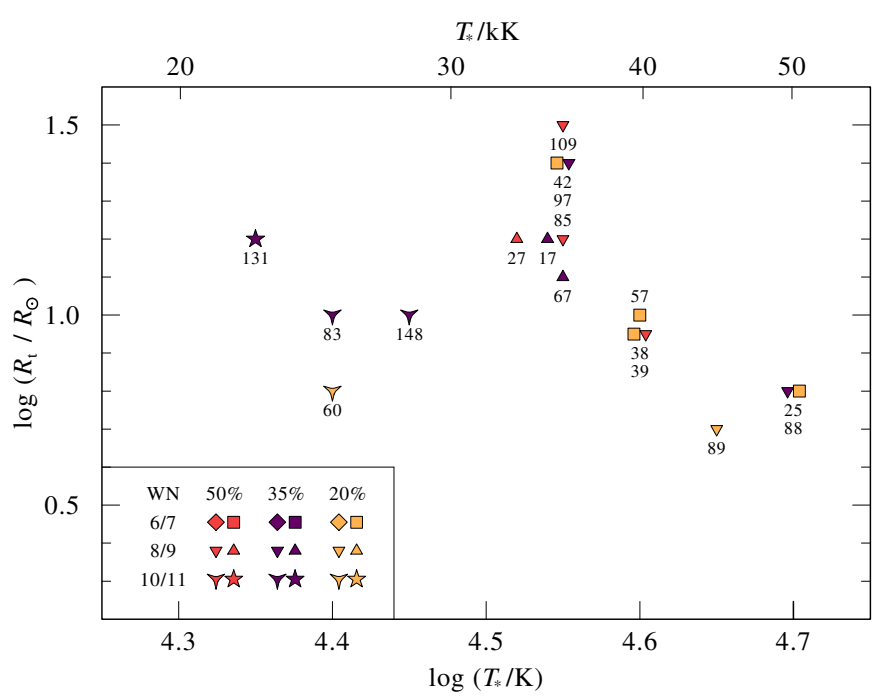

Fig. 4. Obtained positions of the analyzed WNh stars in M 31 in the $\log R_{\mathrm{t}}-\log T_{*}$-plane. The different colors indicate the different hydrogen content. Red: $50 \%$ hydrogen, purple: $35 \%$ hydrogen; orange: $20 \%$ hydrogen. The type of the symbol reflects the WN subtype with a similar meaning as in Fig. 6. The numbers are the M31WR numbers as in Table 2, indicating for which star a particular model has been used.

stage with a helium-burning core. For the stars with higher hydrogen content, one cannot distinguish between younger stars evolving to the red side in the Hertzsprung-Russell diagram and slightly older stars evolving back to the blue. Even stars with $X_{\mathrm{H}} \approx 0.2$ could in principle still be core-hydrogen burning if they were rapid rotators and therefore evolve homogeneously. Ekström et al. (2012) have demonstrated that under this condition, stars with $M_{\text {init }}>60 M_{\odot}$ may still be found on the main sequence, despite having only $20 \%$ of their hydrogen left. Nevertheless, we can discard this scenario as the obtained positions of our WN stars in the Hertzsprung-Russell diagram do not agree with the corresponding evolutionary tracks, which include rotation (see Sect. 5.2).

The empirical mass-loss rates scale with the square root of the adopted clumping density contrast $D$, for which we assume $D=4$ (Hamann \& Koesterke 1998). As expected, our $\dot{M}$ results with clumped WR models are all higher than predicted by the relation for smooth wind O stars from Vink et al. (2000).

\subsection{Hertzsprung-Russell diagram}

The Hertzsprung-Russell diagram of the sample is shown in Fig. 8 with the hydrogen fraction color-coded and the different symbol shapes indicating the WN subtypes. For comparison, the late-type WN stars from the Galaxy (Hamann et al. 2006; Martins et al. 2008; Liermann et al. 2010) and the LMC (Hainich et al. 2014) are plotted. Almost all stars are located on the cool side of the zero-age main sequence.

There are no M 31 stars above $\log L / L_{\odot}=6.0$, in contrast to several Galactic WN stars and at least a few LMC stars. While a part of the luminosities in the Galactic sample might suffer from unsure distances, the sample also includes several stars from the Galactic center region, which is thought to be quite well known in terms of distance. Instead of resembling the Milky Way positions in the HRD, the M 31 results seem to be closer to what we see for the LMC WN population, despite the major differences between these two galaxies.

No star in our sample is from the innermost part of M31, and it is exactly this part of the Milky Way where we find the WN stars with the highest luminosities, such as the so-called "Peony nebula star" WR102ka (Barniske et al. 2008; Oskinova et al. 2013). Therefore the picture might change once we get access to late-type WN stars in this innermost part.

\subsection{Subtype-magnitude relation}

Using the spectral energy distribution from the best-fitting model atmospheres for each star, we can obtain the monochromatic magnitude $M_{v}$ defined by Smith (1968). While the individual value for each object are listed in Table 2, the arithmetic mean and the standard deviation for each subtype are given in Table 3. All stars that entered this calculation contain hydrogen, although the exact amount is different. Due to the problem that the distances are not known for several Galactic WR stars, the only way was to adopt a subtype-magnitude calibration in order to obtain the luminosities.

The results from M 31 now allow us to check the validity of such a calibration for late-type WN stars. Hamann et al. (2006) assumed a brightness magnitude of $M_{v}=-7.22$ mag for all latetype WN stars with hydrogen. With the known distance of M 31, we obtain a mean magnitude of $M_{v}=-6.47$ mag for our sample. However, if we plot the individual $M_{v}$ versus the subtype (Fig. 9), a substantial scatter is encountered. This probably reflects the fact that WN stars do not form a one-parameter sequence, since 
A. Sander et al.: The Wolf-Rayet stars in M31.I.

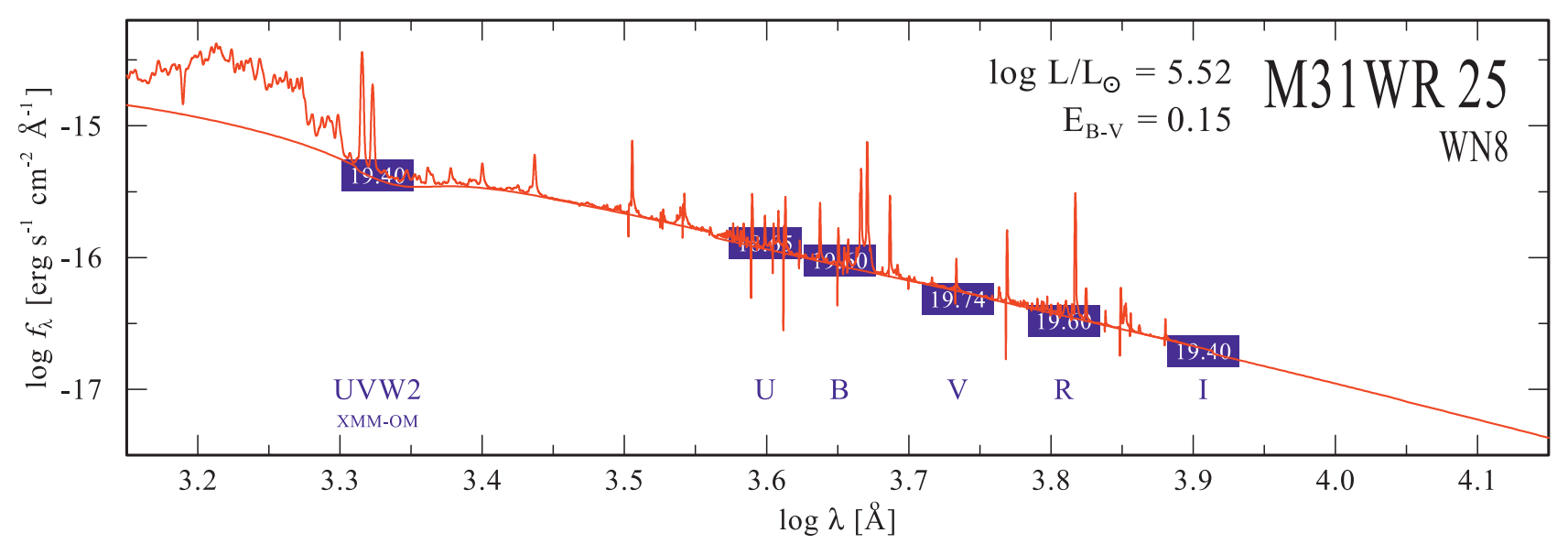

Fig. 5. Spectral energy distribution (SED) of M31WR 25 alias LGGS J004036.76+410104.3 obtained by fitting the observed photometry marks (blue boxes) with the PoWR model (red solid line).

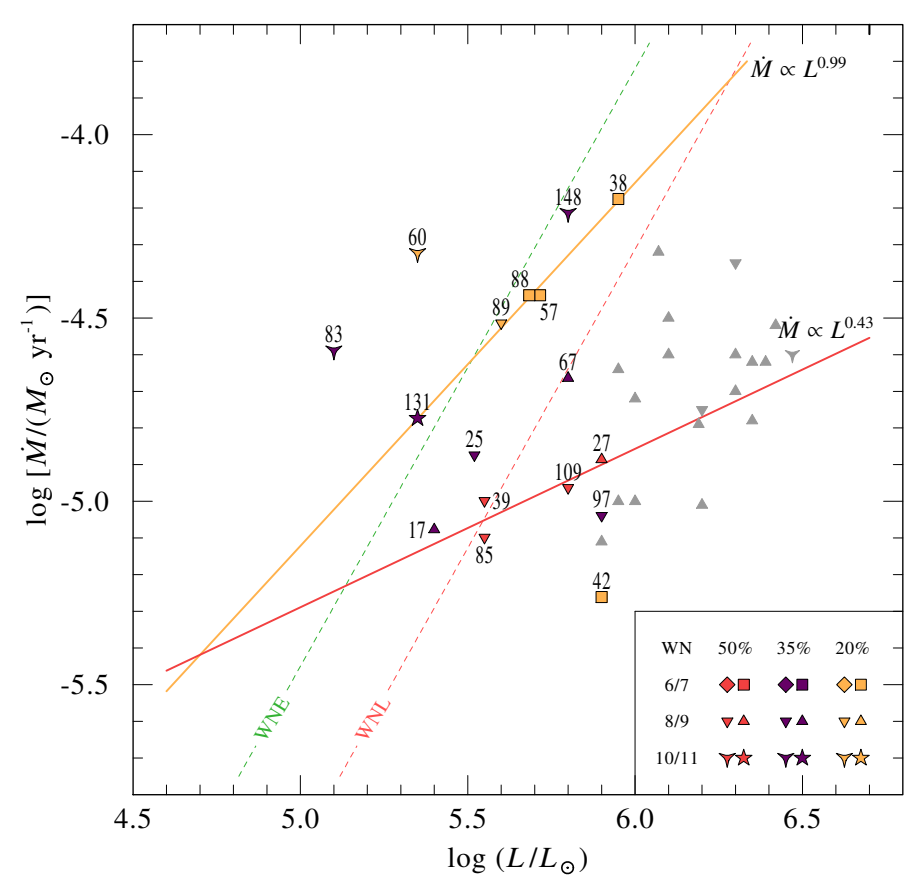

Fig. 6. Empirical mass-loss rate versus luminosity for the M31 latetype WN star sample. The thick lines show the least square fits for the stars with $X_{\mathrm{H}} \approx 0.2$ and $X_{\mathrm{H}} \approx 0.5$, respectively. For comparison, the relations from Nugis \& Lamers (2000) are also shown as dashed lines. The gray symbols are the late-type WN stars near the Galactic center from Martins et al. (2008); Liermann et al. (2010) and Oskinova et al. (2013).

they differ not only in mass, but also in their inner structure. Thus the magnitude calibration cannot work precisely and an uncertainty of about \pm 0.5 mag remains. Moreover, Fig. 9 indicates a systematic trend of $M_{v}$ with subtype rather than a constant value, as reflected by the shown linear regression fit.

After the LMC analyses (Hainich et al. 2014), the present results are a further indicator that at least a part of the Galactic WN stars might be less distant and therefore less luminous than assumed hitherto.

\subsection{Error estimates}

The careful fitting process and the calculation of additional models in those cases where a grid model did not reproduce the

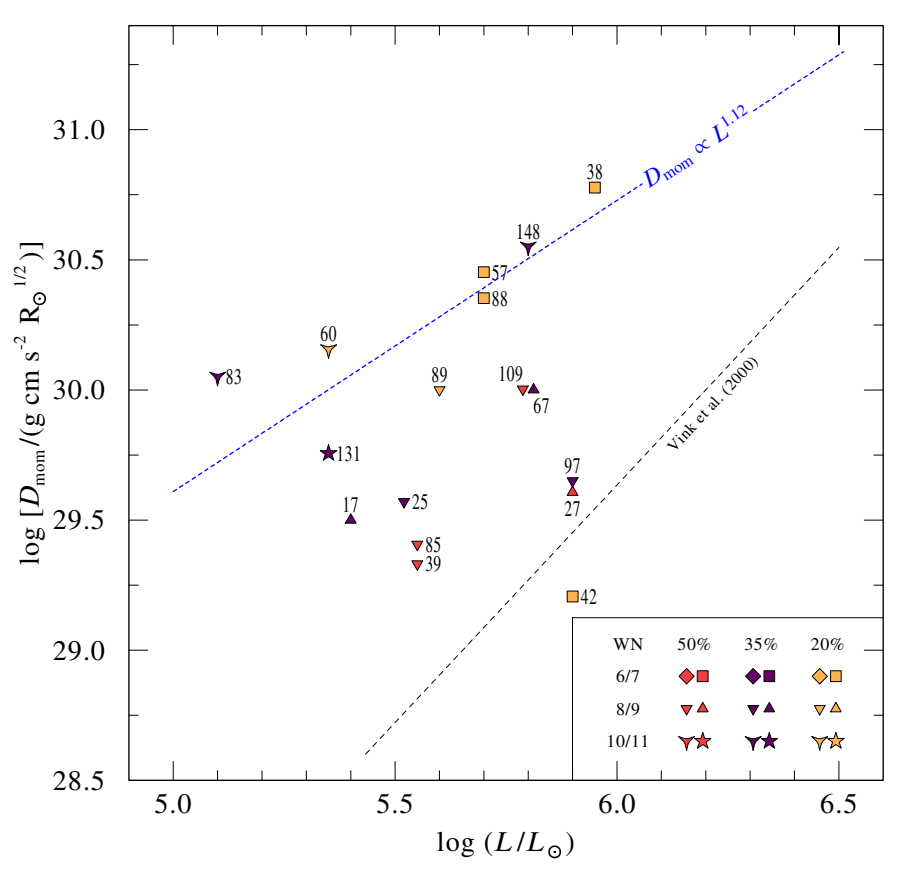

Fig. 7. Modified wind momentum $D_{\text {mom }}$ for the analyzed WN stars, plotted against their luminosities. The blue dotted line shows the least square fit for the stars with $X_{\mathrm{H}} \leq 0.2$. For comparison, the relation from Vink et al. (2000) for O-stars (black dashed line) is also shown.

Table 3. Mean absolute magnitudes per WN subtype in M31.

\begin{tabular}{lcc}
\hline \hline Subtype & $M_{v}[\mathrm{mag}]$ & $\Delta M_{v}[\mathrm{mag}]^{a}$ \\
\hline WN7 & -6.32 & 0.46 \\
WN8 & -6.18 & 0.48 \\
WN9 & -6.58 & 0.57 \\
WN10 & -6.82 & 0.58 \\
WN11 & -6.87 & $0.00^{b}$ \\
\hline
\end{tabular}

Notes. ${ }^{(a)}$ Standard deviation. ${ }^{(b)}$ Only one star available.

observed spectrum well enough meant that the uncertainties in the two main parameters $T_{*}$ and $R_{\mathrm{t}}$ are below one grid cell, i.e., $\leq 0.05 \mathrm{dex}$ in $T_{*}$ and $\leq 0.1 \mathrm{dex}$ in $R_{\mathrm{t}}$. For the terminal velocity $v_{\infty}$ the uncertainty is approximately $25 \%$. The luminosities not only depend on the models, but also on the accuracy of the available photometry, which is only broadband. The distance to M 31 is a 


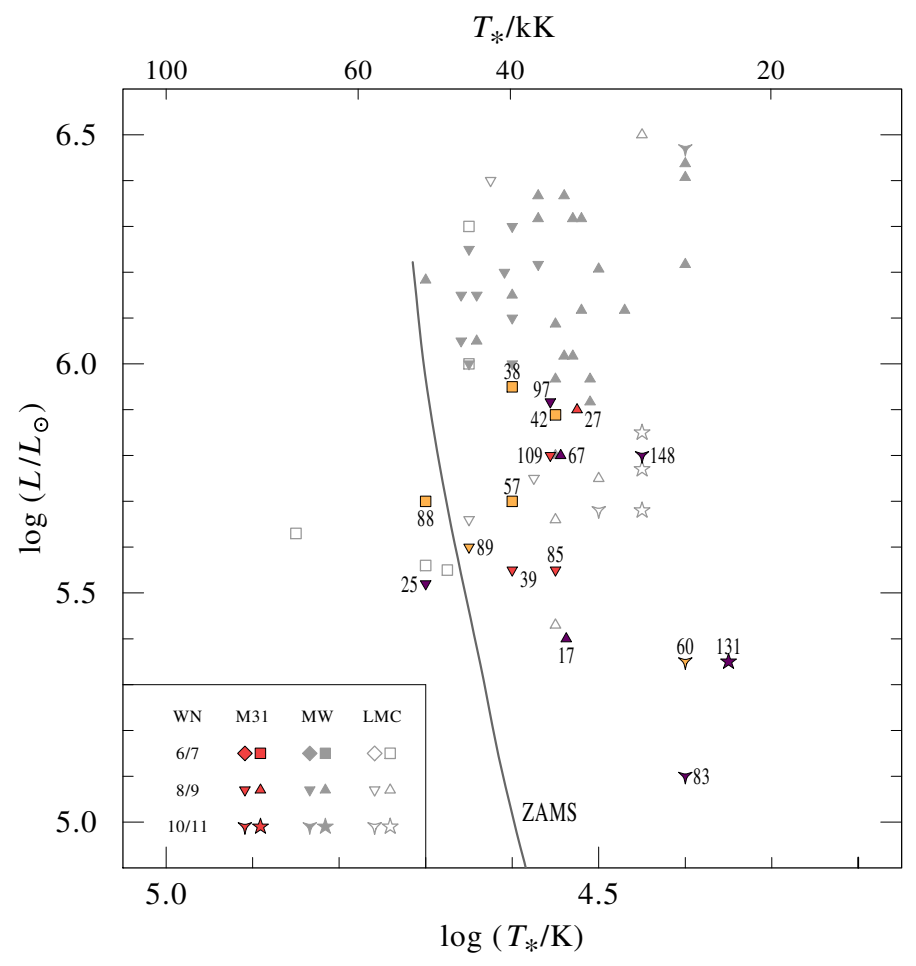

Fig. 8. Hertzsprung-Russell diagram of the M 31 late WN sample. The colored symbols refer to the M31 stars with the colors indicating different hydrogen content as labeled in the previous figures. The filled gray symbols show the positions of the late-type WNh stars from Milky Way using the results from Hamann et al. (2006), Martins et al. (2008), and Liermann et al. (2010). The open gray symbols refer to the LMC late-type WNh stars from Hainich et al. (2014).

minor source of uncertainty. In total, we assume a rather conservative error of 0.1 dex in the stellar luminosity $L$. According to Eq. (1), the uncertainty propagates to 0.15 dex in the mass-loss rate $\dot{M}$. With this value given, the error for the modified wind momentum $D_{\text {mom }}$ is approximately 0.3 dex. These error margins do not include systematic errors from the various assumptions and atomic data used by the stellar atmosphere models that are impossible to quantify.

\section{Discussion of the evolutionary status}

\subsection{Late $W N$ stars as $L B V$ candidates}

The spectra of late WN stars are very similar to those of luminous blue variables (LBVs). In fact, some known LBVs have shown a WN spectral type during their quiet stage, such as AG Car in the late 1980s (Stahl et al. 2001). The term LBV was introduced by Conti (1984) for hot, luminous, and variable stars that do not fit into the WR class, such as $\eta$ Car, P Cyg, and S Dor. The first two of the three stars were already known as variable objects in the 16th century, but it was not until the 1970s when their similarities and their possible connection to a certain stage in the massive star evolution was discussed (see e.g. Humphreys 1975, 1978; Sharov 1975; Humphreys \& Davidson 1979; Wolf et al. 1980).

Today the term LBV is used incoherently in the literature. Although the name might suggest that every luminous and variable blue star could be called an LBV, the original idea was to define this class by a particular type of variability, including phases when the LBV is currently not "blue" (Humphreys \& Davidson 1994). This variability is not theoretically understood,

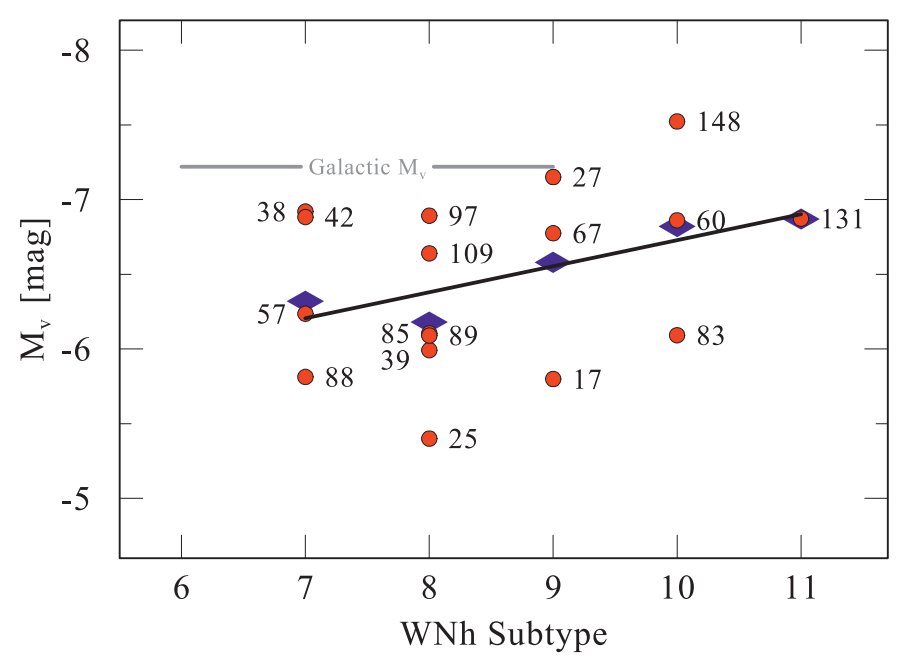

Fig. 9. Mean absolute magnitudes of the WN stars in M31 plotted per subtype. The red dots indicate the positions of the individual stars while the blue diamonds mark the mean value per subtype. The black line shows the linear fit. For comparison, the Galactic relation used in Hamann et al. (2006) is shown in gray.

but observed for several objects and named after the prototypical star S Doradus. In quiescence these stars occupy a certain range in the HR diagram called the S Dor instability strip (Wolf 1989). During "normal outbursts" they brighten by one to two magnitudes in the optical while their bolometric magnitude does not change much.

Combining earlier works such as Humphreys \& Davidson (1994) with newer results, van Genderen (2001) sets up a list of criteria that qualify a star as an LBV candidate. Instead of the vague term LBV he speaks of S Dor (SD) variability and SDmembership which needs to fulfill three criteria, namely photometric variability, visible ejecta, and spectroscopic characteristics that indicate a high luminosity and mass loss, together with the presence of CNO-processed material. Consequently, WN9 to WN11 stars are not automatically considered as LBV candidates unless they show stellar ejecta. If a star has a spectrum that resembles those of a known LBV, and it is known that both brightness and spectral appearance changed significantly on timescales of a few years, it is called a bona fide LBV.

Figure 10 displays the positions of the analyzed stars in the Hertzsprung-Russell diagram (HRD) in comparison to other known LBVs and LBV candidates. In contrast to the version shown in Fig. 8, we now use as the abscissa the effective temperature $T_{\tau=2 / 3}$, which refers to the radius where the Rosseland optical depth reaches two thirds. Remember that $T_{*}$, which is used in Fig. 8, refers to the radius $R_{*}$ at $\tau_{\text {Ross }}=20$, which might be close to the radius of the hydrostatic core. None of the M 31 stars is located beyond the empirical Humphreys-Davidson limit (Humphreys \& Davidson 1979), and only one star (\#148) is in the region of the so-called S Dor instability strip found by Wolf (1989). However, several of our sample stars are not far from this region, while some known LBVs like WRAY 15-751 are close to it, but outside. The bona fide LBV V532, known as Romano's star, even appears as WN8 during its hot phase (Clark et al. 2012; Maryeva \& Abolmasov 2012). We therefore cannot rule out that at least a subsample of our analyzed stars, especially those located at a very similar position as known LBVs, such as \#38 or $\# 27$, could represent LBVs during their quiet stage. In all galaxies where the WR population has been studies comprehensively (MW, LMC, M 31), the very late WN10 and WN11 subtypes are 


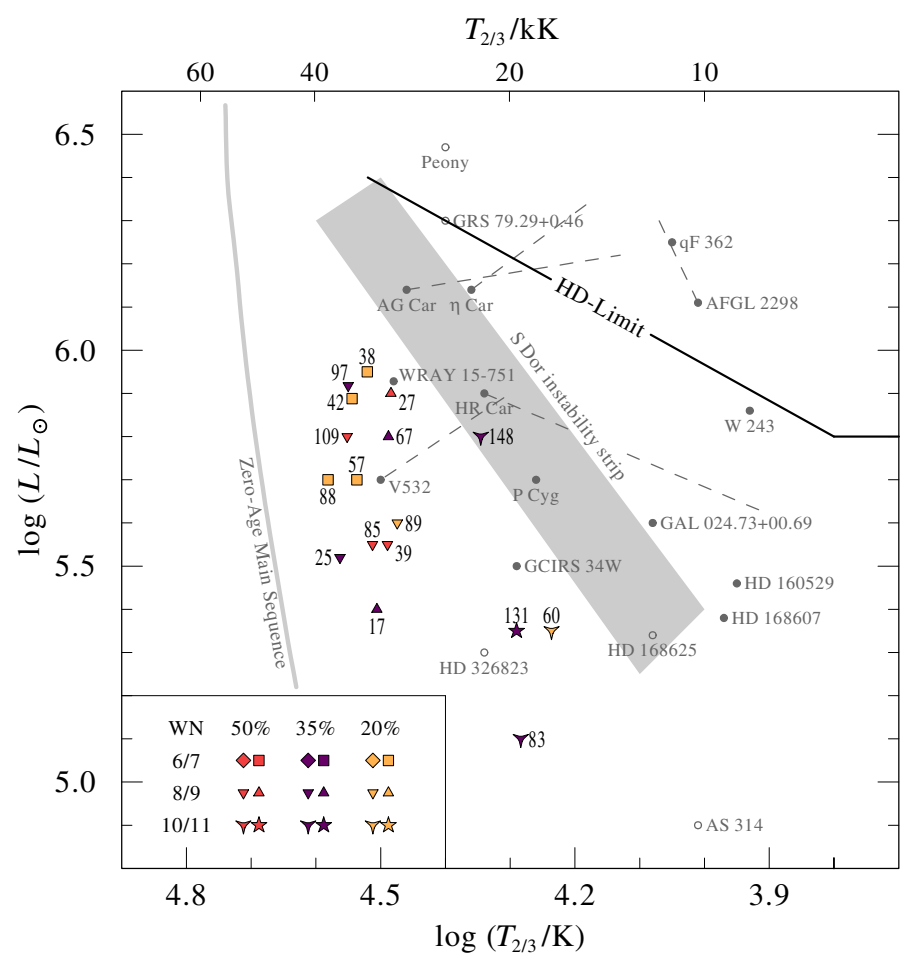

Fig. 10. Hertzsprung-Russell diagram based of the M 31 late-WN sample, this time with $T_{\tau=\frac{2}{3}}$ instead of $T_{*}$. For comparison, known LBVs (filled circles) and LBV candidates (open circles) are shown. The grayshaded area marks the S Dor instability strip (Wolf 1989). The empirical Humphreys-Davidson limit is also indicated. The parameters of the comparison stars are taken from Nazé et al. (2012) with the exception of Romano's Star (V532, Clark et al. 2012; Maryeva \& Abolmasov 2012) and the Peony star (Barniske et al. 2008). The locations of LBVs in different stages are connected by dashed lines. Labels without letters refer to the M31WR numbers as listed in Table 2.

extremely rare. Although there is a certain selection bias in the finding methods as mentioned in Sect.3.1, this might indicate that a star appearing as WN10-11 will not remain in this state for a long time.

One star in our sample, \#60, was mentioned as a PCygnilike LBV candidate by Massey et al. (2007) but later listed as very late WN (WNVL) in Neugent et al. (2012). The star J004334.50+410951.7, classified as Ofpe/WN9 in Massey et al. (2007), does not appear in the M 31 WR census of Neugent et al. (2012).

\subsection{Comparison with evolutionary tracks}

The evolution of massive stars in the upper part of the HRD is still poorly understood. It is not really clear which initial mass ranges lead to the different kinds of WR types and how this depends on the galactic environment and metallicity. Recent studies by Martins \& Palacios (2013) have shown that different evolutionary codes predict significantly different results, especially for hydrogen-rich WN stars above $\log L / L_{\odot}>5.3$.

To discuss the evolutionary situation for the analyzed WN sample, the empirically obtained positions in the HRD are compared to evolutionary tracks. In the past years it has become clear that at least a fraction of the hydrogen-rich WN stars are still core-hydrogen burning (see, e.g., Gräfener et al. 2011). In fact, the most massive stars currently known are hydrogen-rich WN stars (Crowther et al. 2010; Hainich et al. 2014).

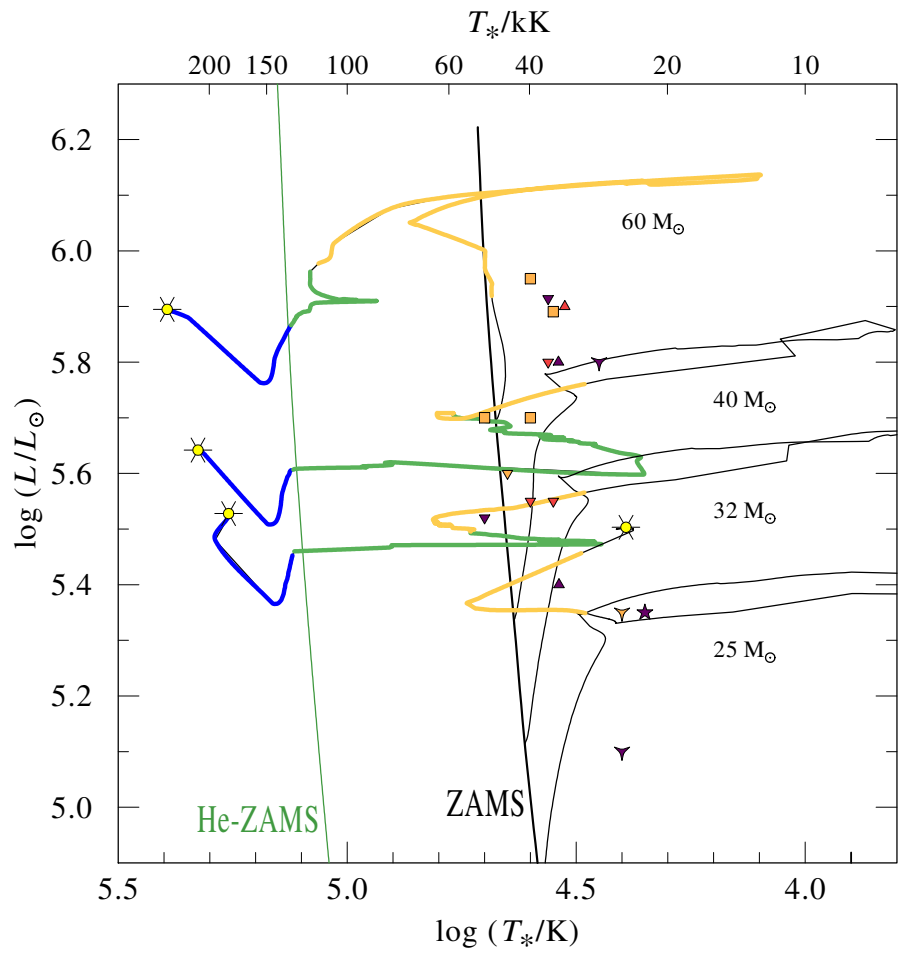

Fig. 11. HR diagram of the M 31 late WN sample compared with the latest set of Geneva evolutionary tracks for $Z=0.014$ (with rotation) from Georgy et al. (2012). The thick orange part of the tracks corresponds to the hydrogen-rich WN phase, while the green and the blue parts indicate the hydrogen-free WN and the WC (incl. WO) phase, respectively.

In Fig. 11, we compare our sample to the current Geneva evolutionary tracks for $Z=0.014$ from Georgy et al. (2012), which include rotation. Based on the surface abundances predicted by the evolutionary models, we highlighted the different WR phases of the tracks using different colors: the hydrogenrich $\left(X_{\mathrm{H}}>0.05\right) \mathrm{WN}$ stage, the hydrogen-free $\left(X_{\mathrm{H}}<0.05\right)$ WN stage, and the WC stage, which we infer from a carbon surface fraction $X_{\mathrm{C}} \geq 0.2$.

For the majority of the stars in our sample there is good agreement with the hydrogen-rich WN phase of the Geneva tracks. However, the tracks cannot explain the low luminosities of the WN11 star \#131 and the two WN10 stars \#60 and \#83. Even if we take into account that some regions of M 31 have a higher metallicity than our Galaxy and therefore compare them with tracks for $Z=0.02$ and $Z=0.03$ from Eldridge et al. (2008) as shown in Figs. 12 and 13, we do not find any track that predicts WR stars at these positions.

Both sets of tracks, the Geneva tracks with rotation and the Eldridge tracks without rotation (Figs. 11 and 12, respectively), fit reasonably well with the HRD positions of the analyzed sample. Apart from the objects mentioned in the previous paragraph, other stars with lower luminosities, such as \#39 or \#75 could be explained either by a $40 M_{\odot}$-track without initial rotation or a $32 M_{\odot}$-track with an initial rotation of $400 \mathrm{~km} \mathrm{~s}^{-1}$. The stars \#60 or \#131 only match the tracks accounting for rotation.

The WN stars analyzed in this paper represent an early stage of the WR evolution where the evolutionary tracks are not very sensitive to details such as rotation. In later stages they differ more. As a result, our forthcoming analyses of the early-type WN stars in M 31 will give us more information about which set of tracks is more adequate. 


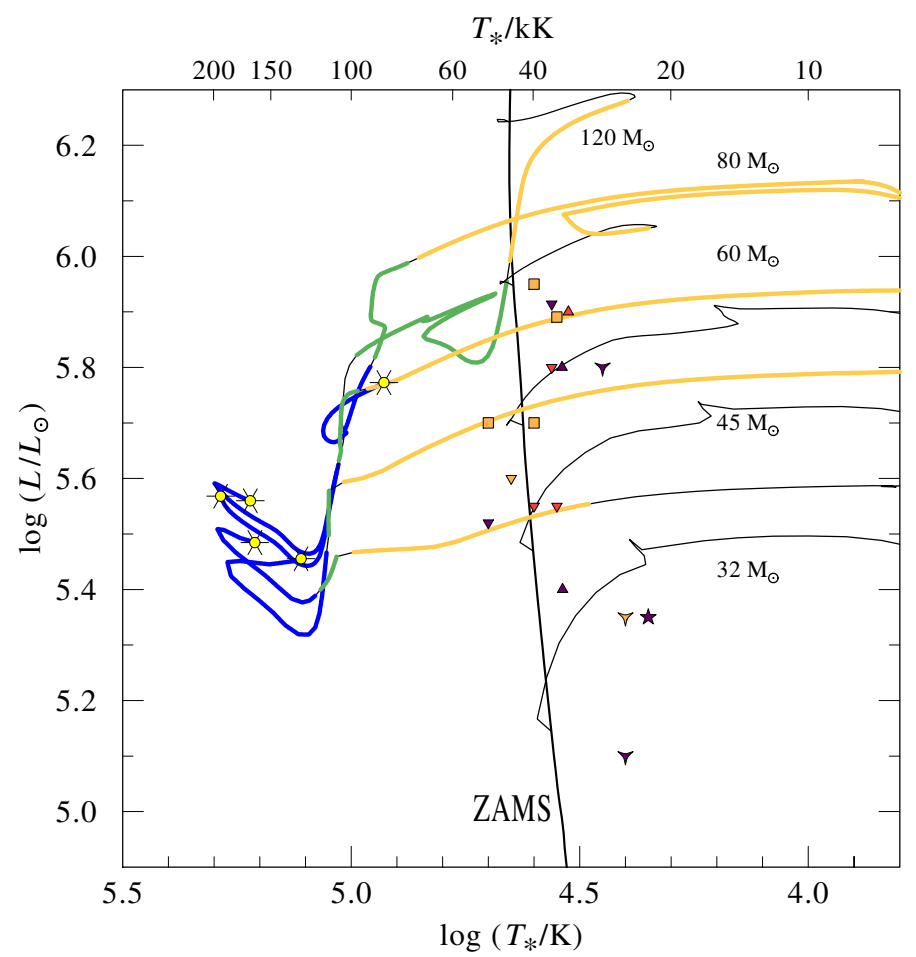

Fig. 12. HR diagram of the M 31 late WN sample compared with the single star evolutionary tracks for $Z=0.02$ from Eldridge et al. (2008). The track colors have the same meaning as in Fig. 11.

So far, we can conclude that the known late-type WN stars in M 31 stem from an initial mass range between 20 and $\approx 60 M_{\odot}$, if formed via single-star evolution. When the objects with the lowest luminosity have undergone binary evolution, the lower limit would rise to 25 or even $30 M_{\odot}$, depending on how many stars are discarded and which set of tracks is appropriate. By using binary models from Eldridge et al. (2013), we can indeed explain the HRD position of \#83, the star with the lowest luminosity in our sample, as the product of binary evolution with an initial mass of $\approx 20 M_{\odot}$.

The highest initial mass in our sample depends even more on the stellar evolution models. The Geneva tracks (Fig. 11) indicate that the most luminous star in our sample (\#38) had an initial mass below $60 M_{\odot}$ while the tracks from Eldridge et al. (2008) for $Z=0.02$ (Fig. 12) would indicate an initial mass above $60 M_{\odot}$. The tracks for $Z=0.03$ (Fig. 13) would increase this value even to $80 M_{\odot}$, but this higher metallicity is not supported by our spectral fits, as is evident from the good match of the nitrogen lines (cf. Fig. A.4).

For none of the WR stars in M31, we obtained a luminosity of $\log L / L_{\odot}>6.0$, which would indicate a very massive star. Interestingly, this situation does not seem to be restricted to WR stars. A current work from Humphreys et al. (2013) dealing with luminous and variable stars does not list any star in M31 above this limit either.

However, the most massive stars so far have been found in very massive clusters or special environments, such as the central region of our Galaxy or R136 in the LMC. It might just be a selection effect that such stars have not been identified M 31 so far. On the other hand, if there really are no very massive stars in M31, this would match with the low SFR calculated by Neugent et al. (2012).

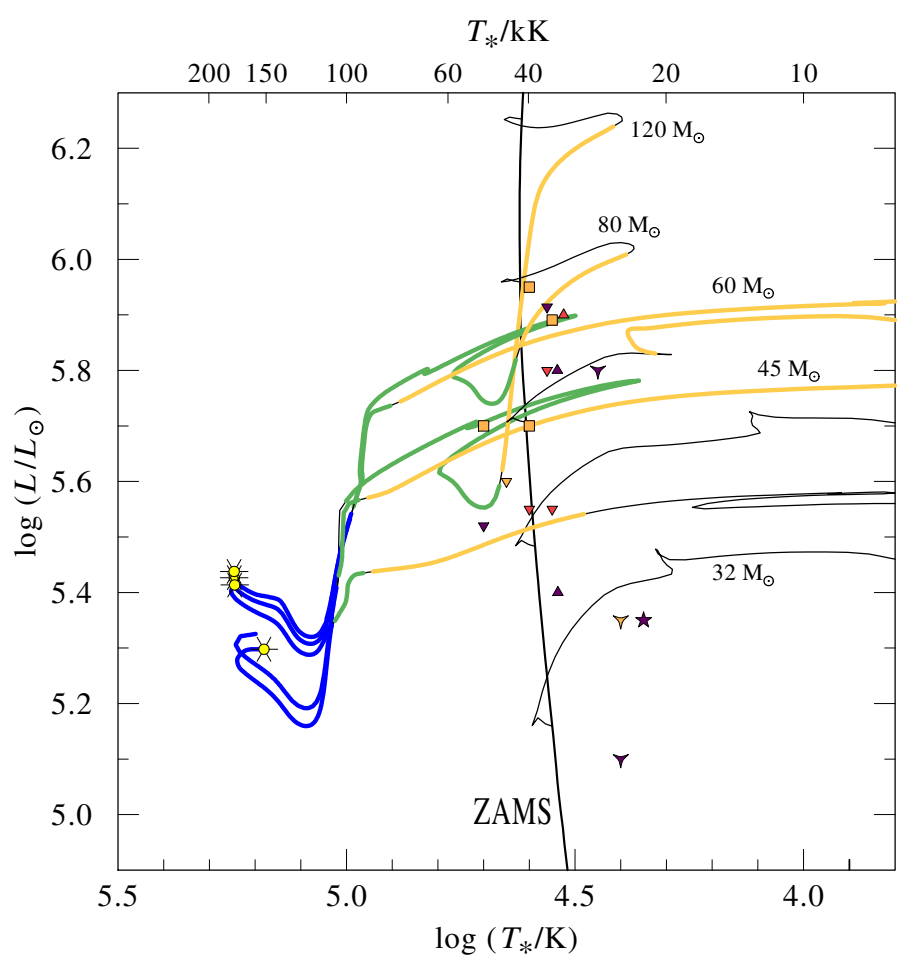

Fig. 13. Same as Fig. 12, but for $Z=0.03$.

\section{Conclusions}

For the first time, we have analyzed a complete sample of latetype WN stars in a Milky-Way-like galaxy. We analyzed 17 latetype WN stars in the Andromeda Galaxy (M31), including all known WN7 to WN11 stars with available spectra of sufficient quality. With the known distance of M 31, we can avoid the large uncertainty in the luminosities of Galactic WR stars. We draw the following conclusions:

- All stars in our sample have luminosities $L$ between $10^{5}$ and $10^{6} L_{\odot}$. Notably, we do not find any star in our sample that exceeds $10^{6} L_{\odot}$.

- The absolute visual magnitude $M_{\mathrm{V}}$ shows a significant scatter, even within the same WN subtype. This sets limits to the applicability of a subtype magnitude calibration.

- Since the current catalog of WN stars in M31 does not include the central region of this galaxy, we cannot rule out that this special environment hosts WN stars with luminosities $L>10^{6} L_{\odot}$, especially since we know that such stars exist in the LMC and the Galactic center region.

- The late-type WN stars in M31 are in good agreement with the latest set of the Geneva tracks with $Z=0.014$. Only the Geneva tracks with rotation can reproduce the lowest luminosities in our sample. Otherwise, the different sets of tracks (Georgy et al. 2012; Eldridge et al. 2008) can all explain the observed WNh stars because these stars are not yet in the final WR stages for which the tracks differ more.

- If formed via single-star evolution, the analyzed late-type WN stars in M31 stem from an initial mass range between 20 and $60 M_{\odot}$. The lower limit might in fact be higher if the few stars with the lowest luminosities were formed via binary evolution.

- Our spectral fits reveal that the analyzed late-type WN stars have similar chemical compositions to their Galactic counterparts. This is in line with the fact that the stars are not located in the inner part of M31, where we would expect 
higher metallicities. The obtained HRD positions of the analyzed stars do not provide additional constraints since they can be reproduced by evolutionary models with both Galactic and higher metallicity.

- The number of very late WN stars (WN9-11) is low, which could partly be a selection effect of the detection method (Neugent et al. 2012). While a lot of additional WN9 stars have been discovered in the Milky Way in obscured regions with infrared observations, the number of currently known WN10 and WN11 stars in M31 is about the same as in the Milky Way and the LMC.

- Only one object, the WN10 star \#148, is located in the S Dor instability strip in the HR-diagram. However, the HRD positions do not rule out that some of the stars of very late-type WN subtypes might be LBVs in the quiescent stage. From the luminosity range, it is hard to tell whether the WN stars have lost parts of their hydrogen via an LBV outbursts or in the red supergiant (RSG) phase.

In a forthcoming paper we will analyze the early WN subtypes to get a more complete picture of the WN population in M 31 .

Acknowledgements. We would like to thank the referee, Cyril Georgy, for his constructive feedback that improved the present work. This research has made use of the SIMBAD database and the VizieR catalog access tool, both operated at the CDS, Strasbourg, France. The Digitized Sky Surveys were produced at the Space Telescope Science Institute under U.S. Government grant NAG W-2166. The images of these surveys are based on photographic data obtained using the Oschin Schmidt Telescope on Palomar Mountain and the UK Schmidt Telescope. The plates were processed into the present compressed digital form with the permission of these institutions. We acknowledge the use of NASA's SkyView facility located at NASA Goddard Space Flight Center. The first author of this work (A.S.) is supported by the Deutsche Forschungsgemeinschaft (DFG) under grant HA 1455/22.

\section{References}

Barbá, R. H., Gamen, R., Arias, J. I., et al. 2010, in Rev. Mex. Astron. Astrofis. Conf. Ser., 38, 30

Barniske, A., Oskinova, L. M., \& Hamann, W.-R. 2008, A\&A, 486, 971

Clark, J. S., Castro, N., Garcia, M., et al. 2012, A\&A, 541, A146

Conti, P. S. 1984, in Observational Tests of the Stellar Evolution Theory, eds. A. Maeder \& A. Renzini, IAU Symp., 105, 233

Crowther, P. A., Hillier, D. J., \& Smith, L. J. 1995, A\&A, 293, 172

Crowther, P. A., Schnurr, O., Hirschi, R., et al. 2010, MNRAS, 408, 731

Davies, B., Clark, J. S., Trombley, C., et al. 2012, MNRAS, 419, 1871

Ekström, S., Georgy, C., Eggenberger, P., et al. 2012, A\&A, 537, A146
Eldridge, J. J., Izzard, R. G., \& Tout, C. A. 2008, MNRAS, 384, 1109

Eldridge, J. J., Fraser, M., Smartt, S. J., Maund, J. R., \& Crockett, R. M. 2013, MNRAS, 436, 774

Esteban, C., \& Peimbert, M. 1995, in Rev. Mex. Astron. Astrofis. Conf. Ser. 3, eds. M. Pena, \& S. Kurtz, 133

Fitzpatrick, E. L. 1999, PASP, 111, 63

Georgy, C., Ekström, S., Meynet, G., et al. 2012, A\&A, 542, A29

Gräfener, G., Koesterke, L., \& Hamann, W. 2002, A\&A, 387, 244

Gräfener, G., Vink, J. S., de Koter, A., \& Langer, N. 2011, A\&A, 535, A56

Greenawalt, B., Walterbos, R. A. M., \& Braun, R. 1997, ApJ, 483, 666

Hainich, R., Rühling, U., Todt, H., et al. 2014, A\&A, accepted [arXiv: 1401.5474]

Hamann, W., \& Gräfener, G. 2004, A\&A, 427, 697

Hamann, W., \& Koesterke, L. 1998, A\&A, 335, 1003

Hamann, W., Gräfener, G., \& Liermann, A. 2006, A\&A, 457, 1015

Humphreys, R. M. 1975, ApJ, 200, 426

Humphreys, R. M. 1978, ApJ, 219, 445

Humphreys, R. M., \& Davidson, K. 1979, ApJ, 232, 409

Humphreys, R. M., \& Davidson, K. 1994, PASP, 106, 1025

Humphreys, R. M., Davidson, K., Grammer, S., et al. 2013, ApJ, 773, 46

Karachentsev, I. D., Karachentseva, V. E., Huchtmeier, W. K., \& Makarov, D. I. 2004, AJ, 127, 2031

Krabbe, A., Genzel, R., Eckart, A., et al. 1995, ApJ, 447, L95

Liermann, A., Hamann, W.-R., Oskinova, L. M., Todt, H., \& Butler, K. 2010 , A\&A, 524, A82

Martins, F., \& Palacios, A. 2013, A\&A, 560, A16

Martins, F., Hillier, D. J., Paumard, T., et al. 2008, A\&A, 478, 219

Maryeva, O., \& Abolmasov, P. 2012, MNRAS, 419, 1455

Massey, P., Olsen, K. A. G., Hodge, P. W., et al. 2006, AJ, 131, 2478

Massey, P., McNeill, R. T., Olsen, K. A. G., et al. 2007, AJ, 134, 2474

Nazé, Y., Rauw, G., \& Hutsemékers, D. 2012, A\&A, 538, A47

Neugent, K. F., Massey, P., \& Georgy, C. 2012, ApJ, 759, 11

Nugis, T., \& Lamers, H. J. G. L. M. 2000, A\&A, 360, 227

Oskinova, L. M., Hamann, W.-R., \& Feldmeier, A. 2007, A\&A, 476, 1331

Oskinova, L. M., Steinke, M., Hamann, W.-R., et al. 2013, MNRAS, 436, 3357

Page, M. J., Brindle, C., Talavera, A., et al. 2012, MNRAS, 426, 903

Rubin, V. C., \& Ford, Jr., W. K. 1970, ApJ, 159, 379

Sana, H., de Koter, A., de Mink, S. E., et al. 2013, A\&A, 550, A107

Sander, A., Hamann, W.-R., \& Todt, H. 2012, A\&A, 540, A144

Schmutz, W., Hamann, W., \& Wessolowski, U. 1989, A\&A, 210, 236

Sharov, A. S. 1975, in Variable Stars and Stellar Evolution, eds. V. E. Sherwood, \& L. Plaut, IAU Symp., 67, 275

Smith, L. F. 1968, MNRAS, 140, 409

Stahl, O., Jankovics, I., Kovács, J., et al. 2001, A\&A, 375, 54

van den Bergh, S. 1964, ApJS, 9, 65

van der Hucht, K. A. 2001, New Astron. Rev., 45, 135

van Genderen, A. M. 2001, A\&A, 366, 508

Vanbeveren, D., \& Conti, P. S. 1980, A\&A, 88, 230

Vink, J. S., de Koter, A., \& Lamers, H. J. G. L. M. 2000, A\&A, 362, 295

Wolf, B. 1989, A\&A, 217, 87

Wolf, B., Appenzeller, I., \& Cassatella, A. 1980, A\&A, 88, 15

Zaritsky, D., Kennicutt, Jr., R. C., \& Huchra, J. P. 1994, ApJ, 420, 87 


\section{Appendix A: Spectral fits}

Table A.1. Appendix overview: analyzed late-type WN stars in M31.

\begin{tabular}{lcccc}
\hline \hline M31WR & LGGS & WR type & Figure & Page \\
\hline 17 & J004024.33+405016.2 & WN9 & A.1 & 13 \\
25 & J004036.76+410104.3 & WN8 & A.2 & 13 \\
27 & J004056.49+410308.7 & WN9 & A.3 & 14 \\
38 & J004126.11+411220.0 & WN7 & A.4 & 14 \\
39 & J004126.39+411203.5 & WN8 & A.5 & 15 \\
42 & J004130.37+410500.9 & WN7-8 & A.6 & 15 \\
57 & J004238.90+410002.0 & WN7 & A.7 & 16 \\
60 & J004242.33+413922.7 & WN10 & A.8 & 16 \\
67 & J004302.05+413746.7 & WN9 & A.9 & 17 \\
83 & J004337.10+414237.1 & WN10 & A.10 & 17 \\
85 & J004344.48+411142.0 & WN8 & A.11 & 18 \\
88 & J004353.34+414638.9 & WN7 & A.12 & 18 \\
89 & J004357.31+414846.2 & WN8 & A.13 & 19 \\
97 & J004413.06+411920.5 & WN8 & A.14 & 19 \\
109 & J004430.04+415237.1 & WN8 & A.15 & 20 \\
131 & J004511.21+420521.7 & WN11 & A.16 & 20 \\
148 & J004542.26+414510.1 & WN10 & A.17 & 21 \\
\hline
\end{tabular}


A. Sander et al.: The Wolf-Rayet stars in M31. I.
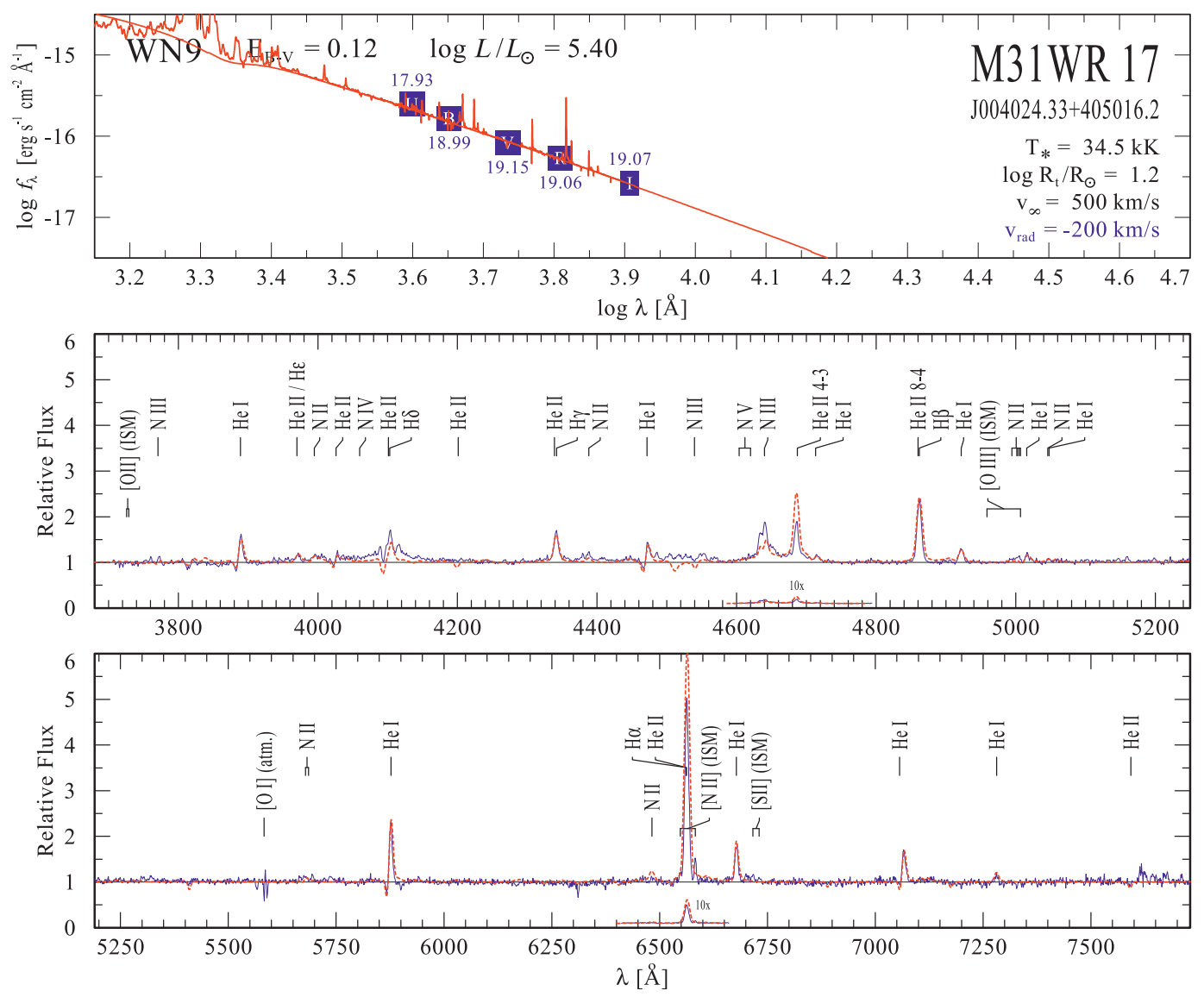

Fig. A.1. Spectral fit for M31WR 17.
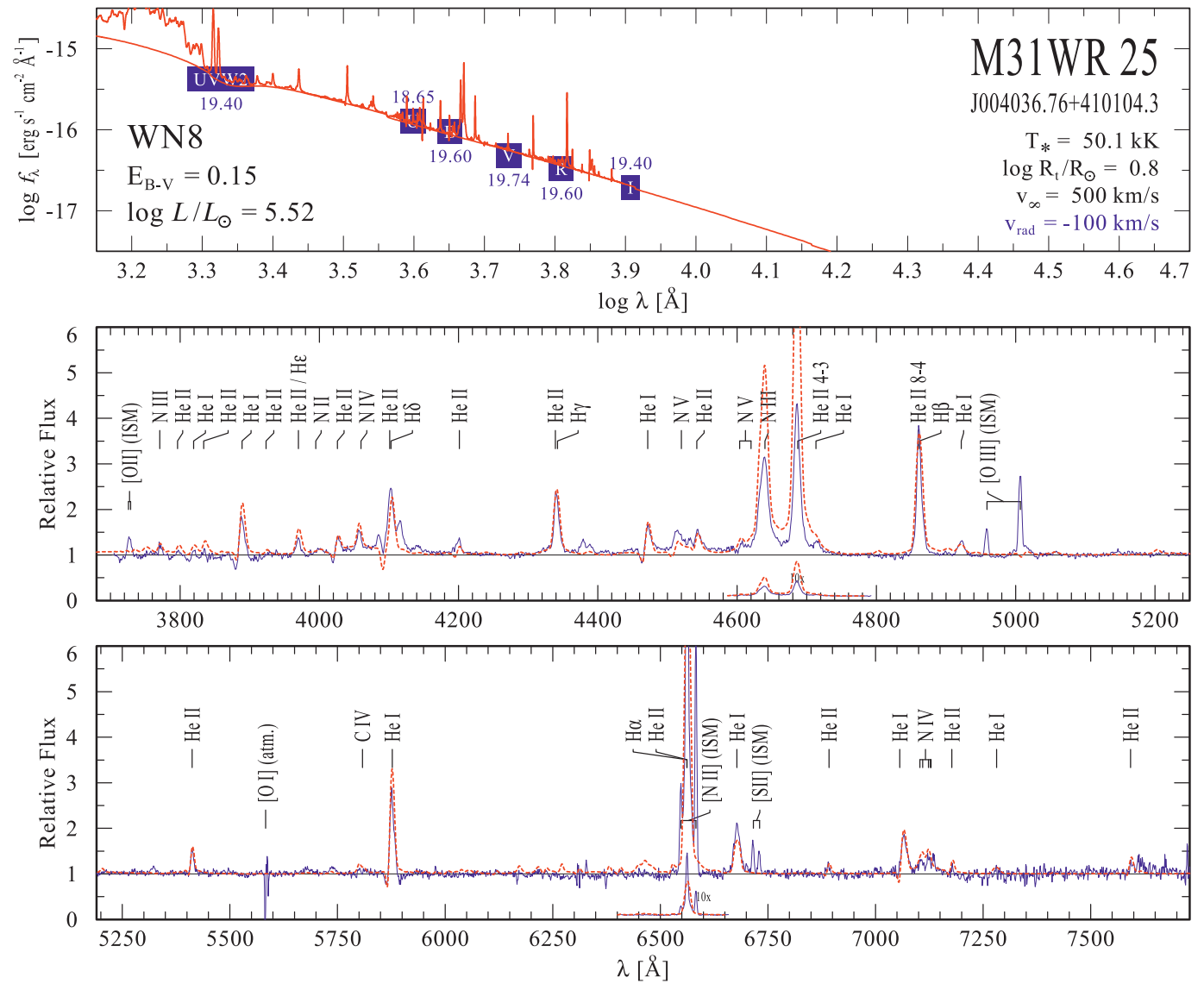

Fig. A.2. Spectral fit for M31WR 25. 
A\&A 563, A89 (2014)
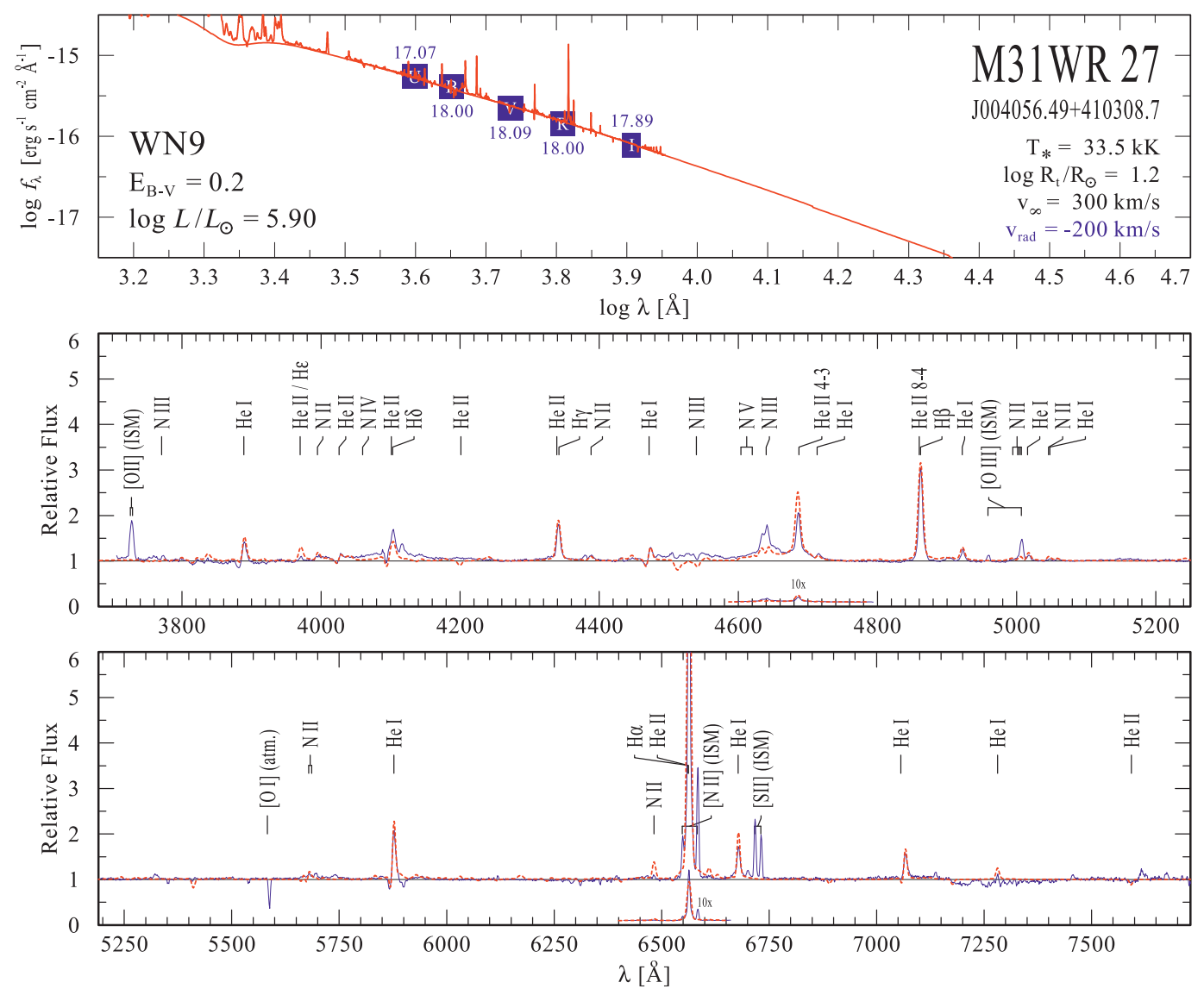

Fig. A.3. Spectral fit for M31WR 27.
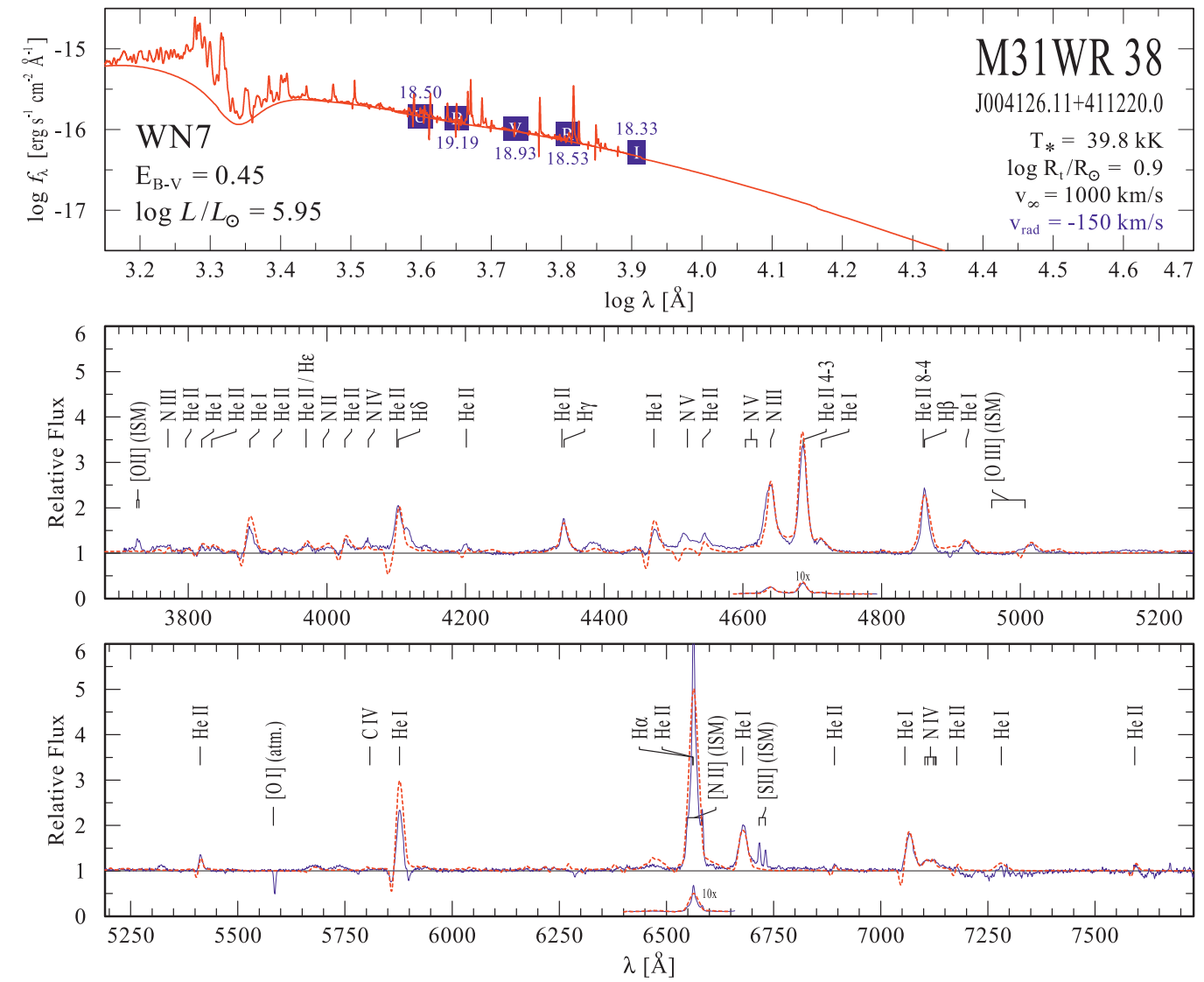

Fig. A.4. Spectral fit for M31WR 38. 
A. Sander et al.: The Wolf-Rayet stars in M31. I.
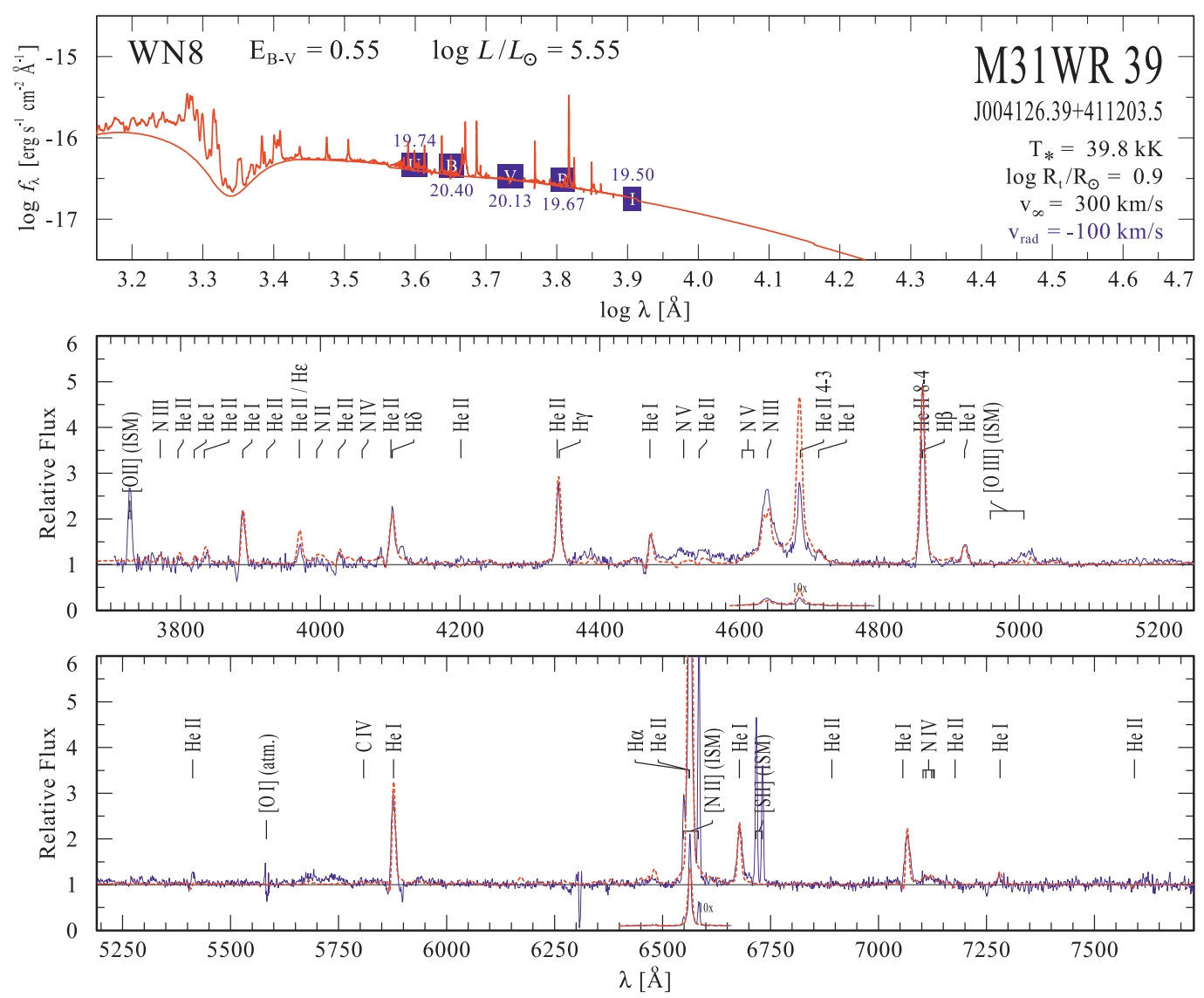

Fig. A.5. Spectral fit for M31WR 39.
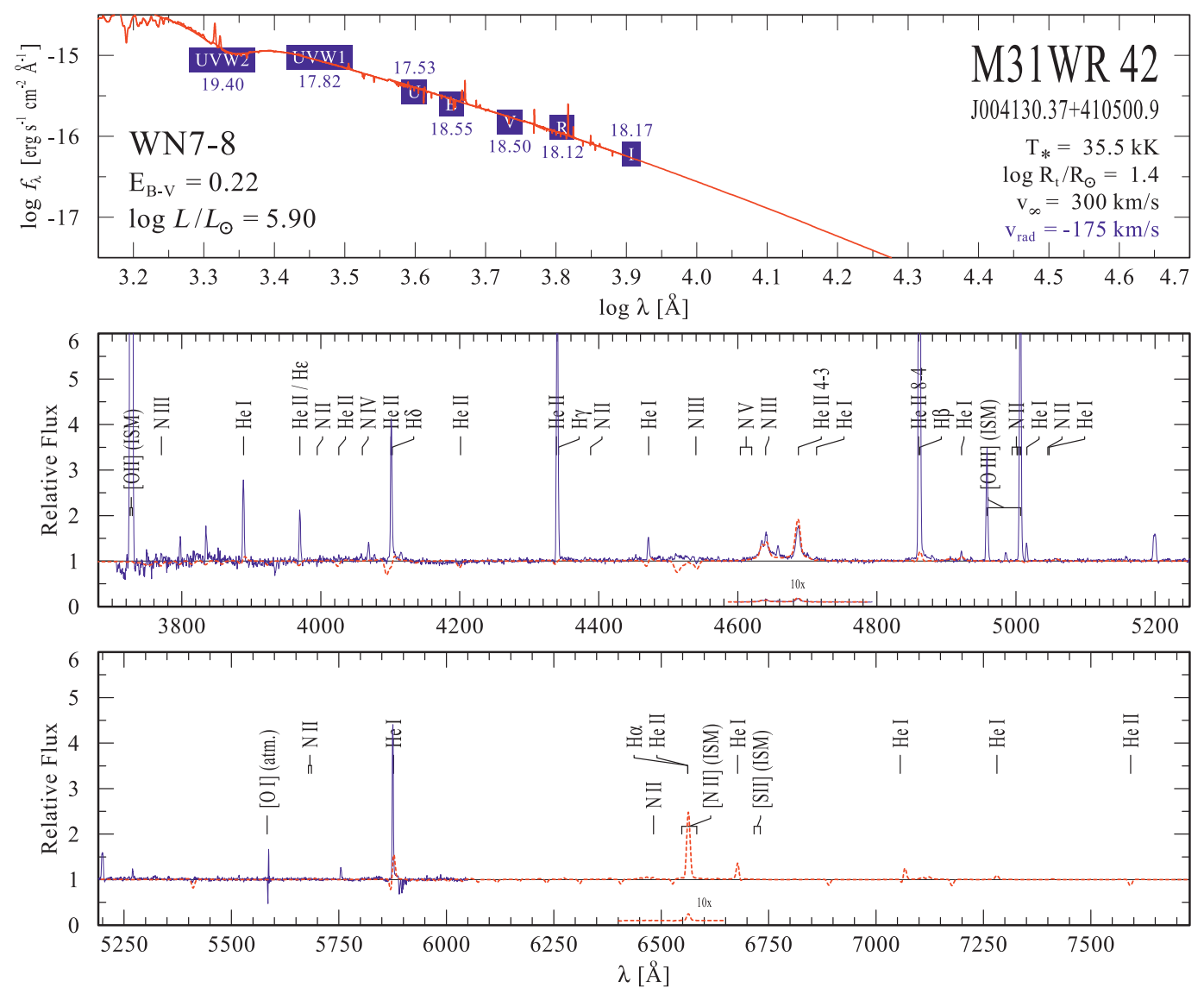

Fig. A.6. Spectral fit for M31WR 42. 

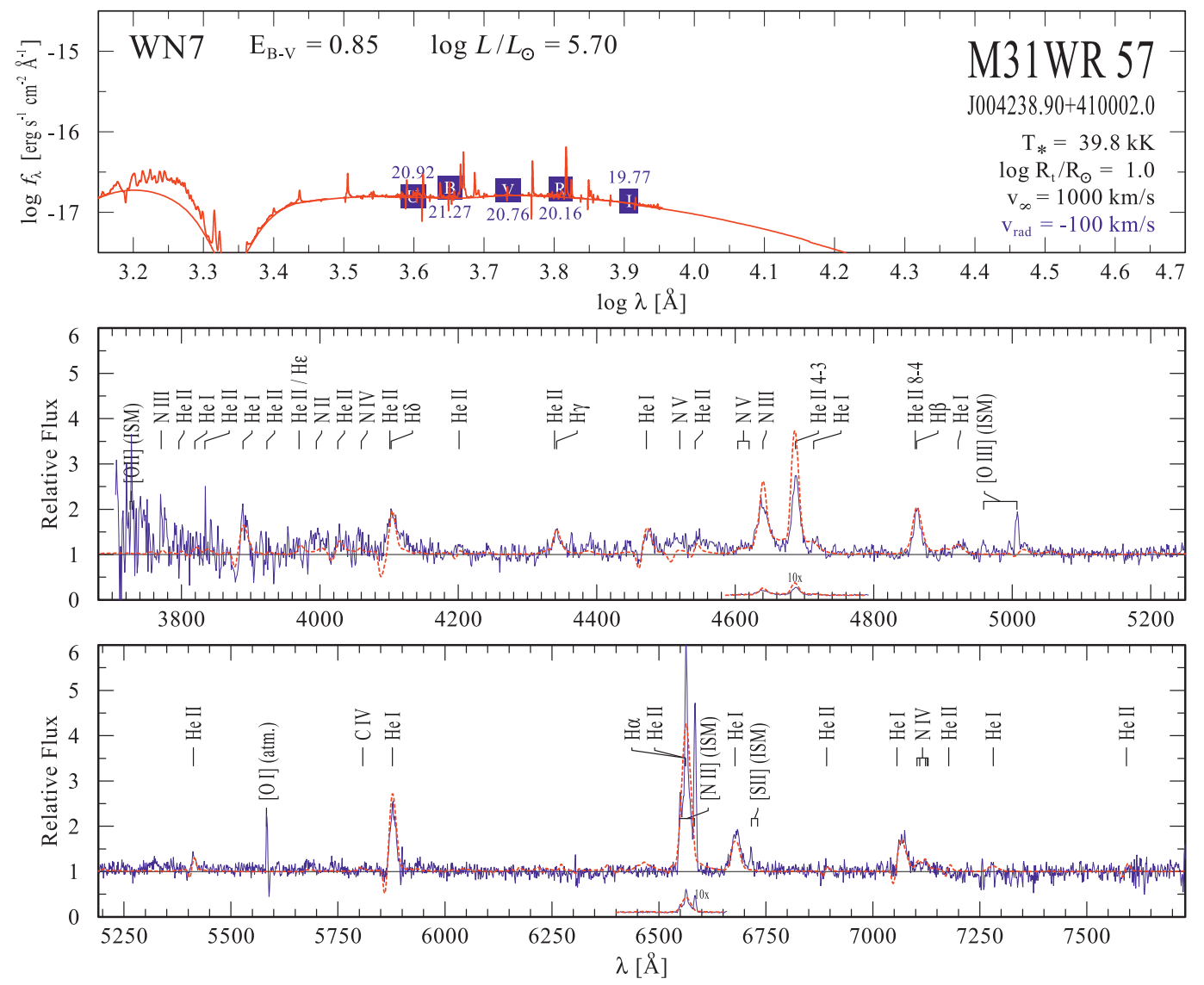

Fig. A.7. Spectral fit for M31WR 57.
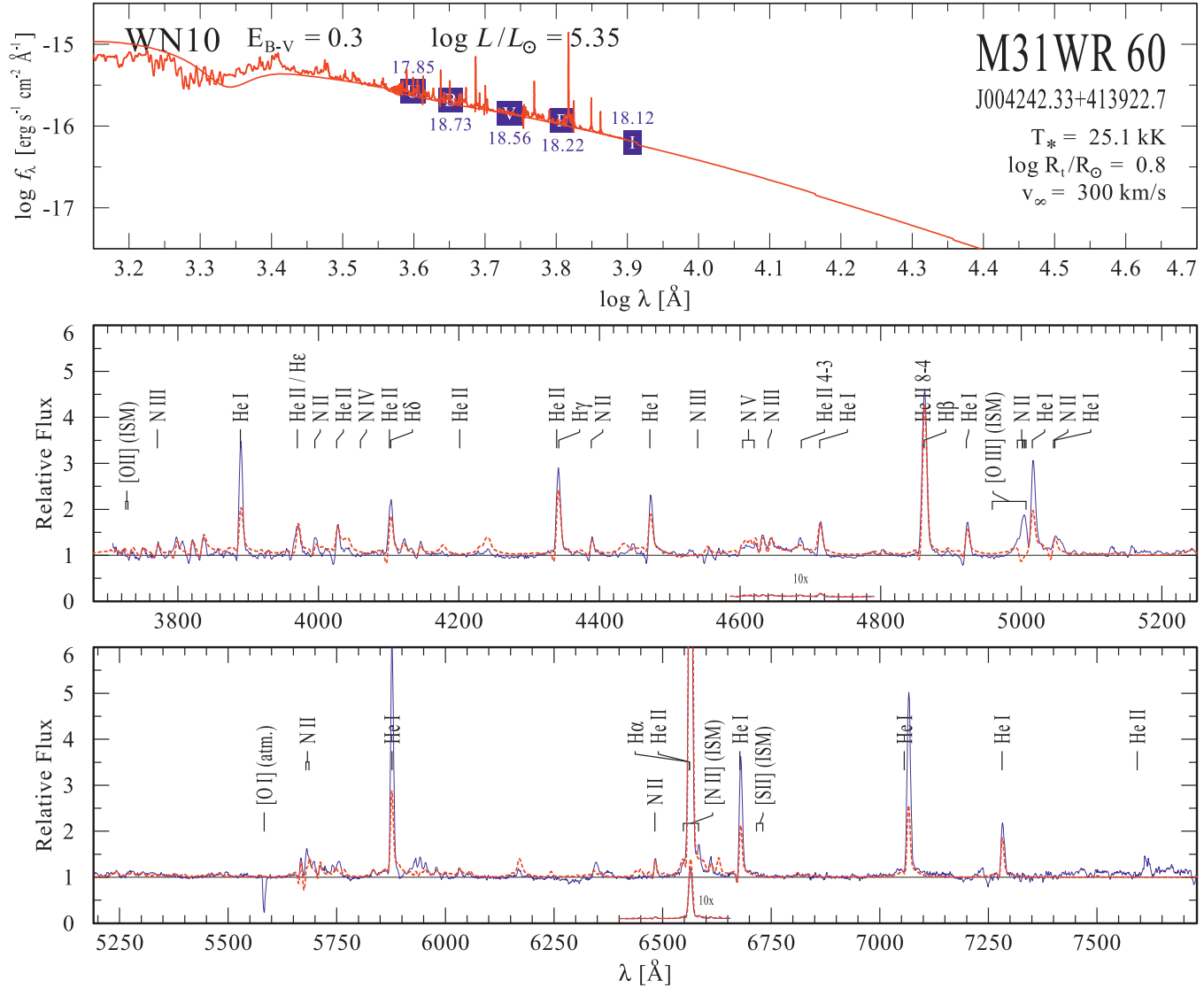

Fig. A.8. Spectral fit for M31WR 60. 
A. Sander et al.: The Wolf-Rayet stars in M31. I.
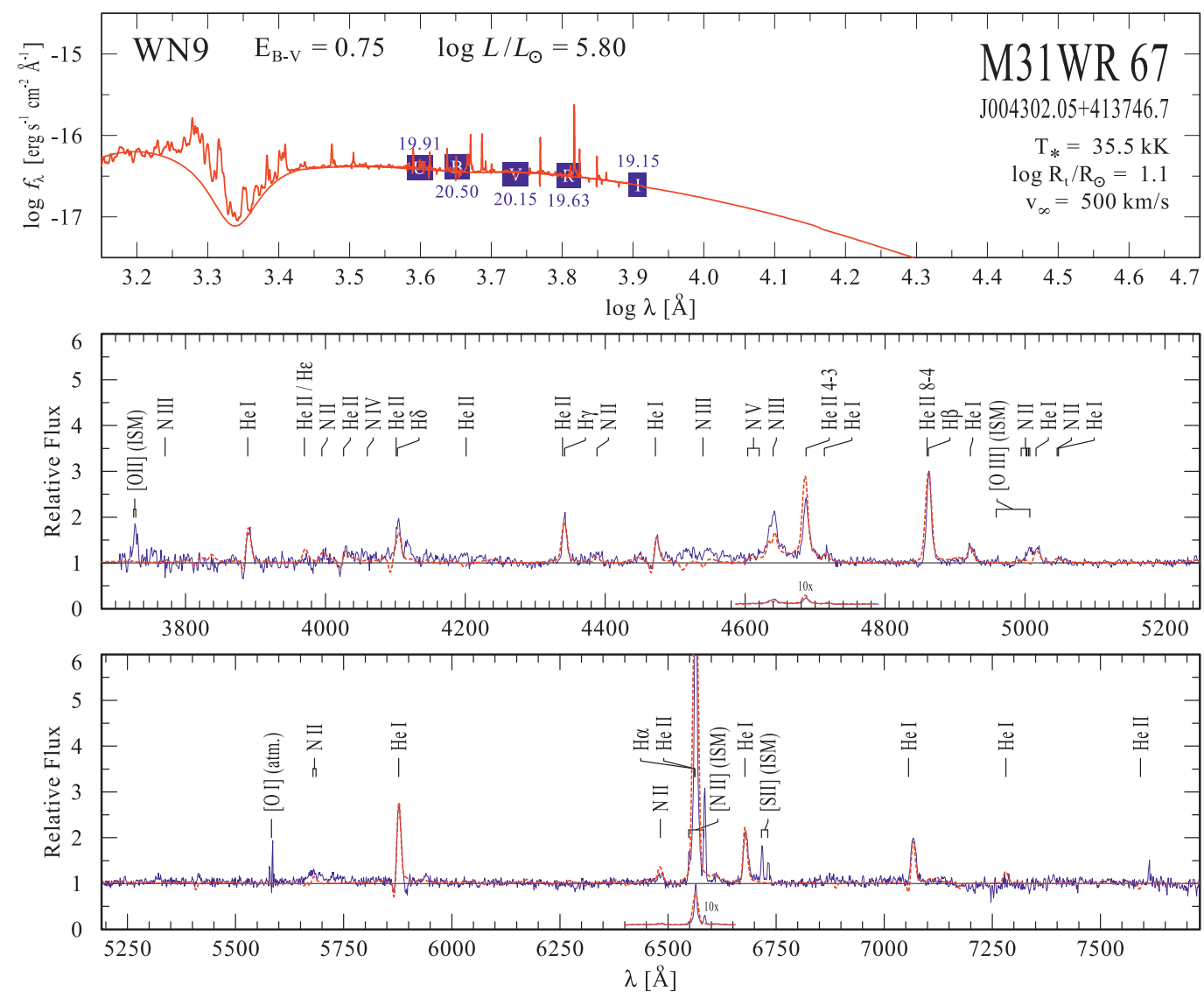

Fig. A.9. Spectral fit for M31WR 67.
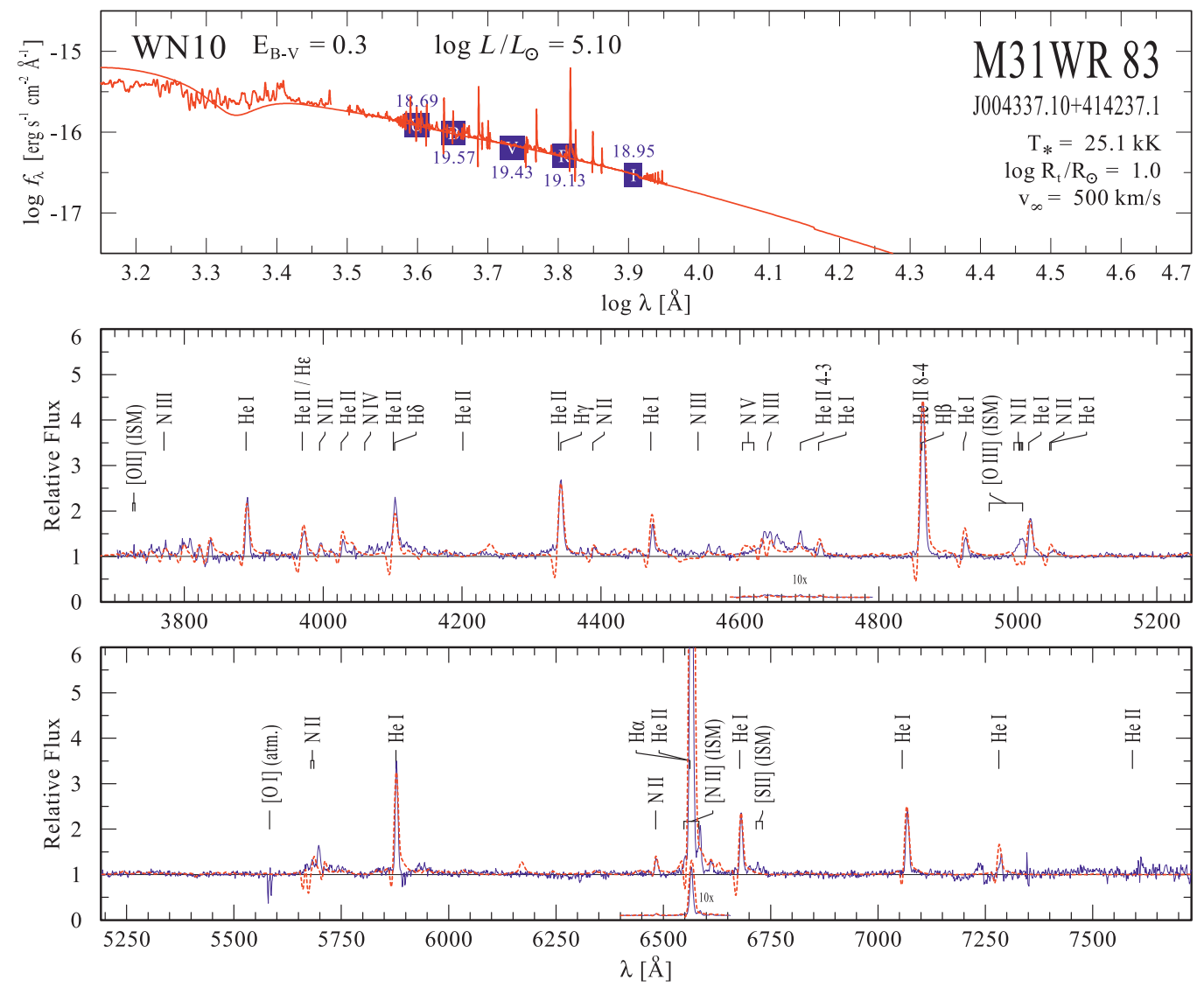

Fig. A.10. Spectral fit for M31WR 83. 
A\&A 563, A89 (2014)
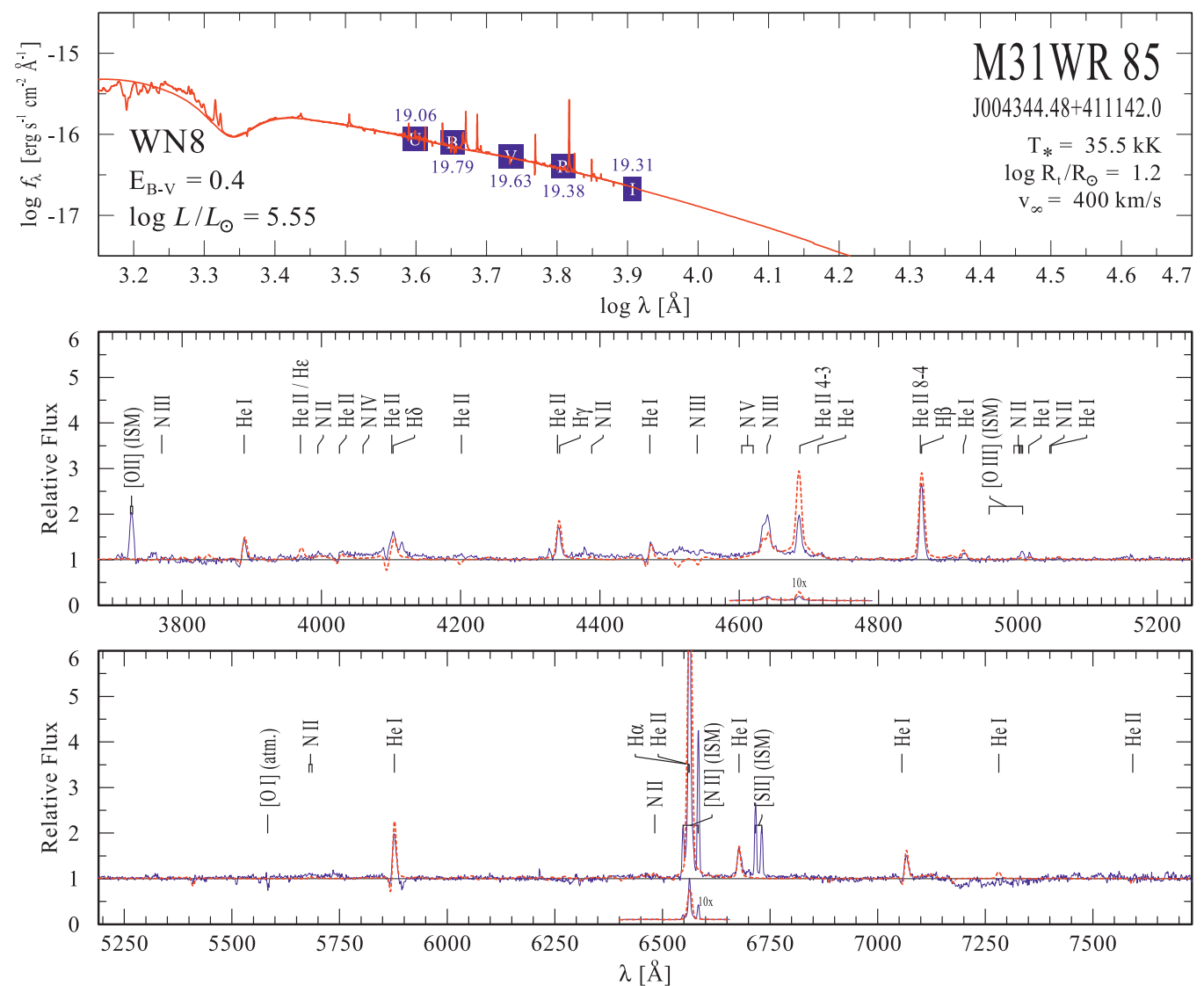

Fig. A.11. Spectral fit for M31WR 85.
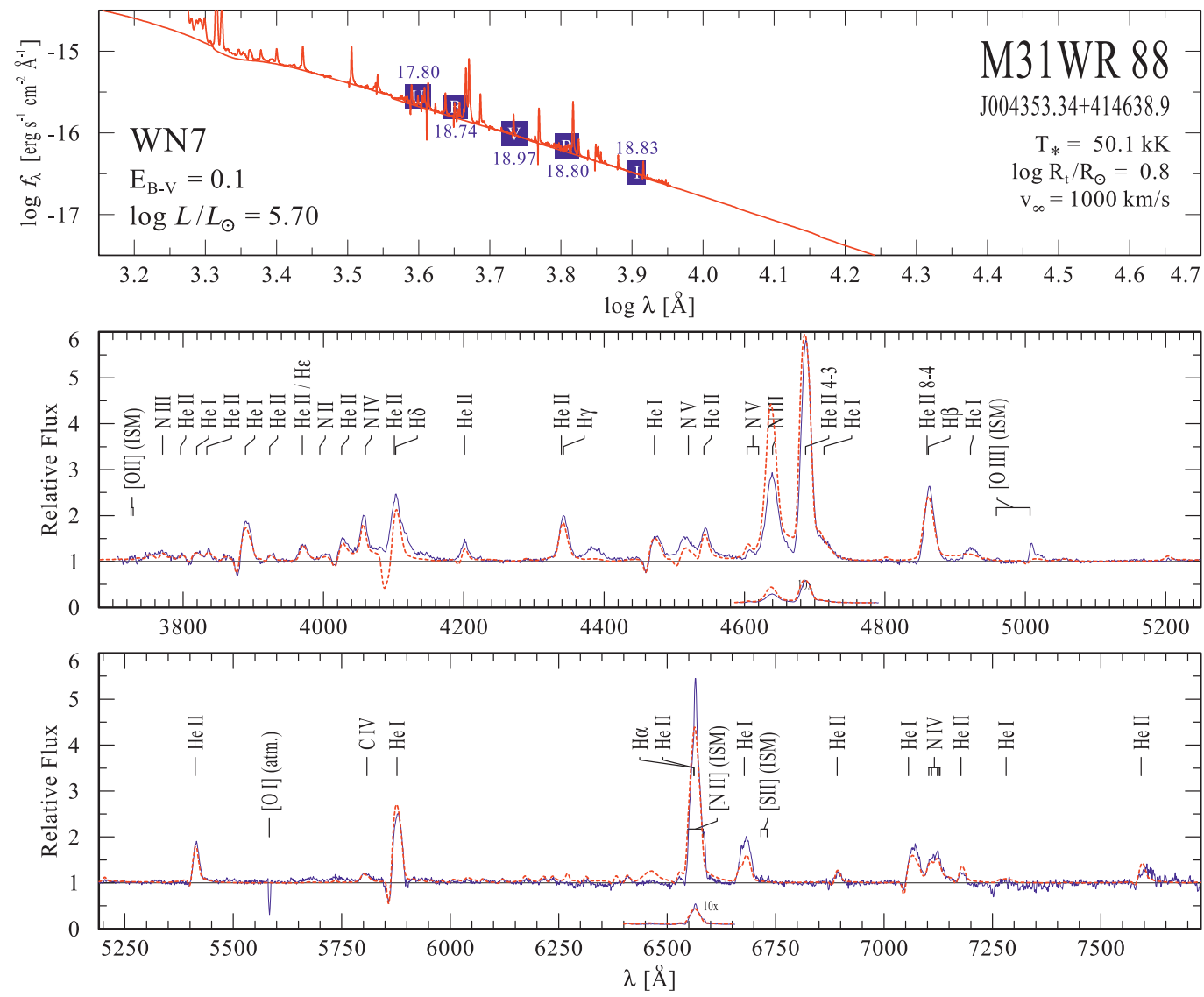

Fig. A.12. Spectral fit for M31WR 88. 
A. Sander et al.: The Wolf-Rayet stars in M31. I.
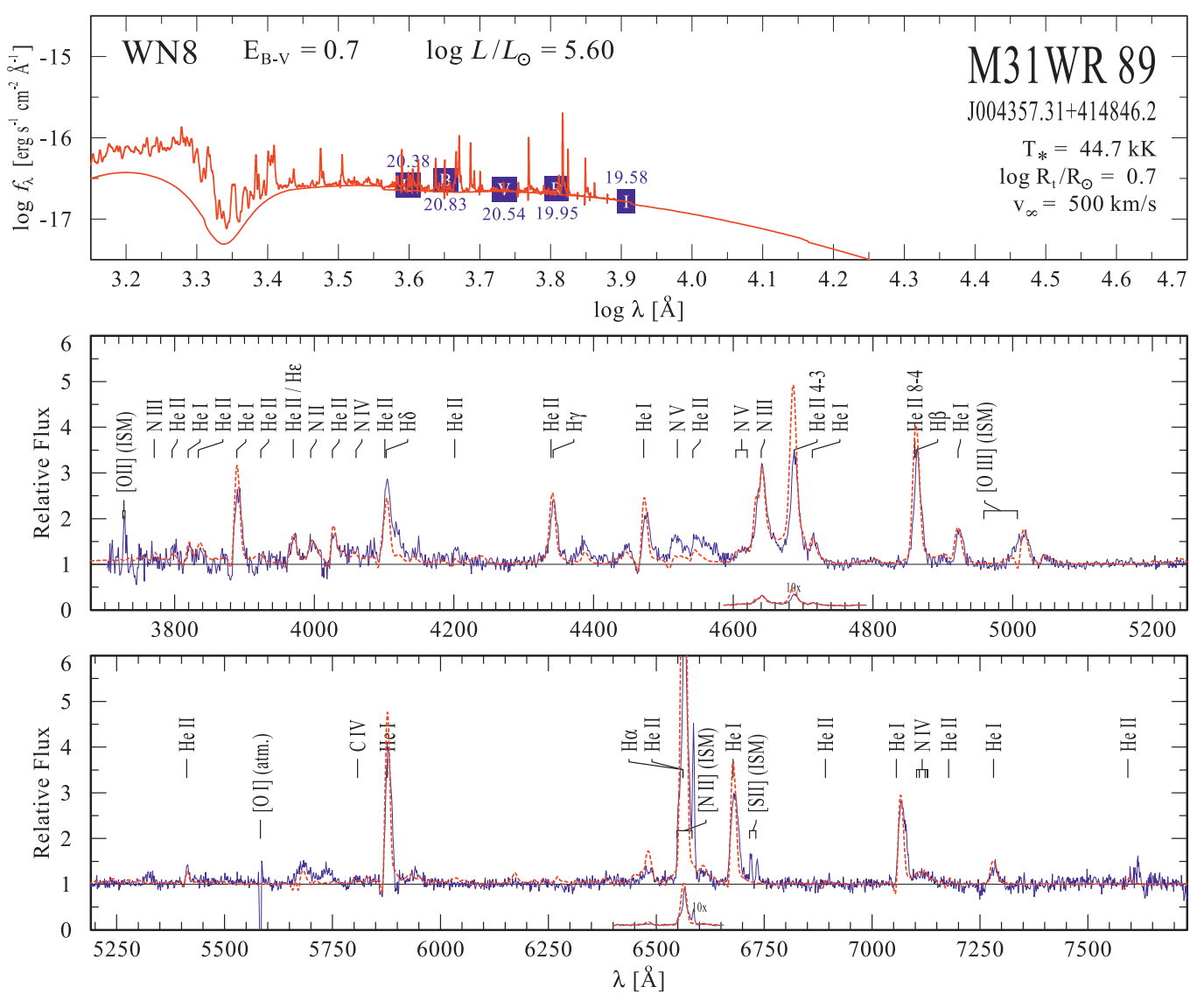

Fig. A.13. Spectral fit for M31WR 89.
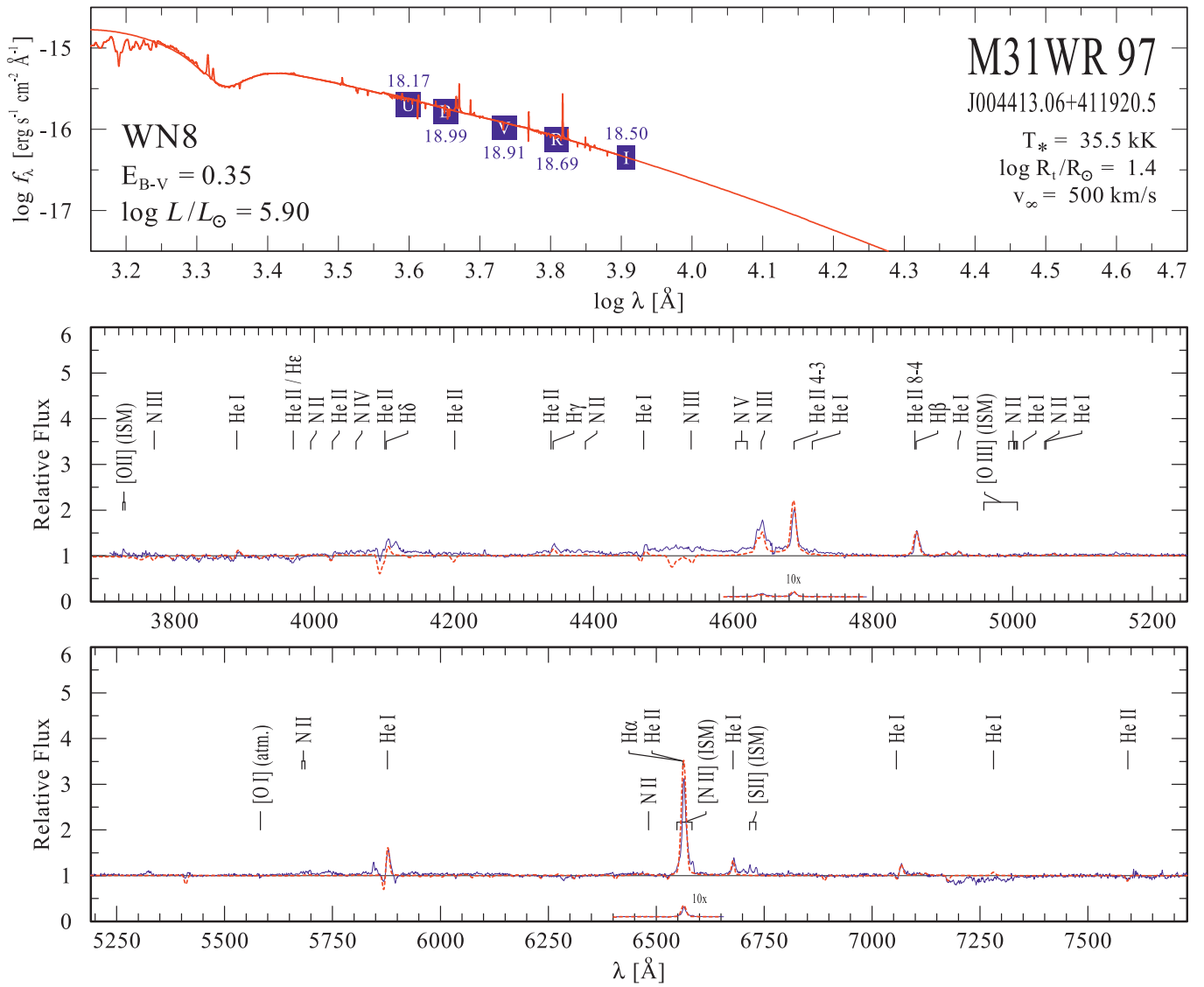

Fig. A.14. Spectral fit for M31WR 97. 

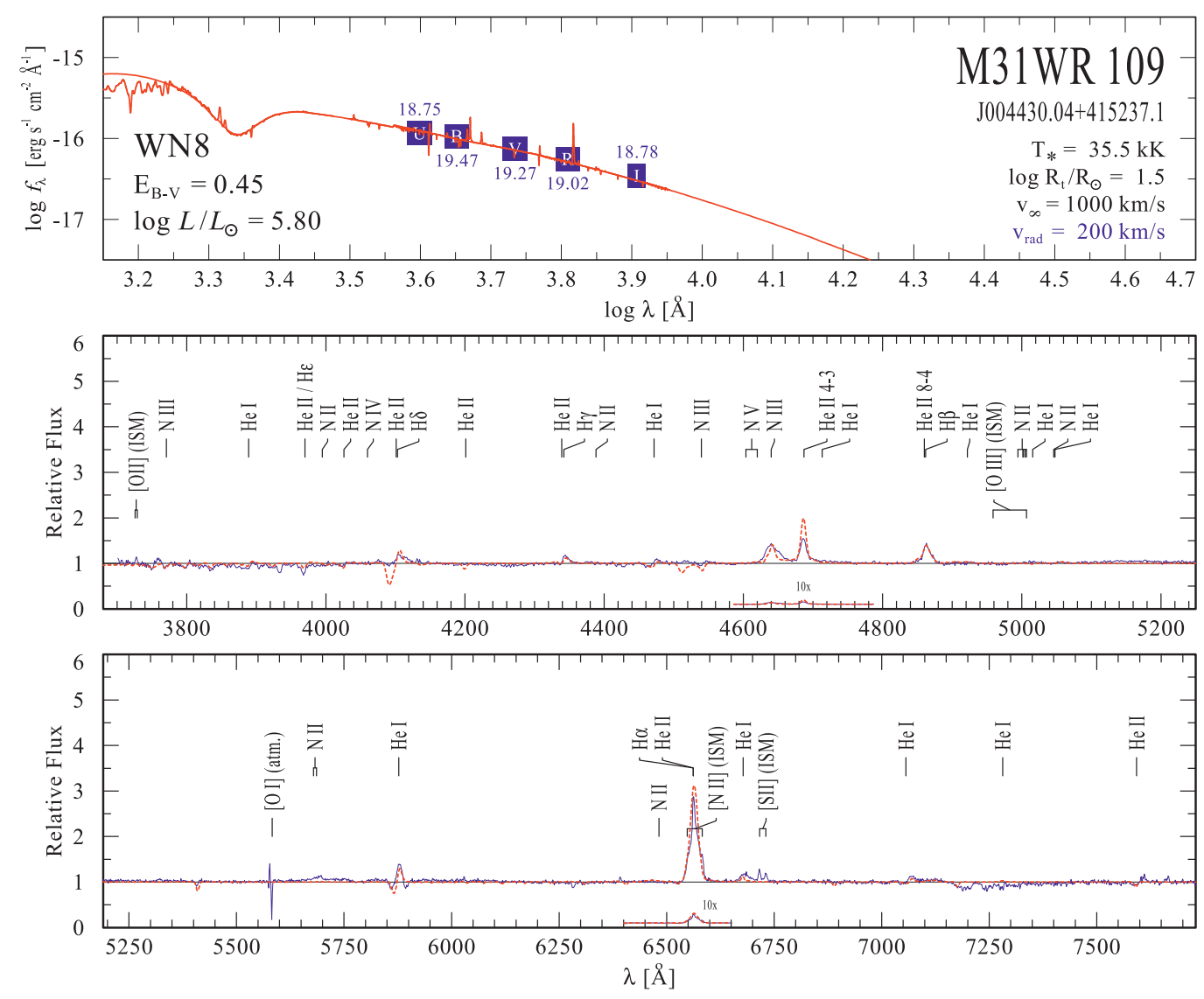

Fig. A.15. Spectral fit for M31WR 109.
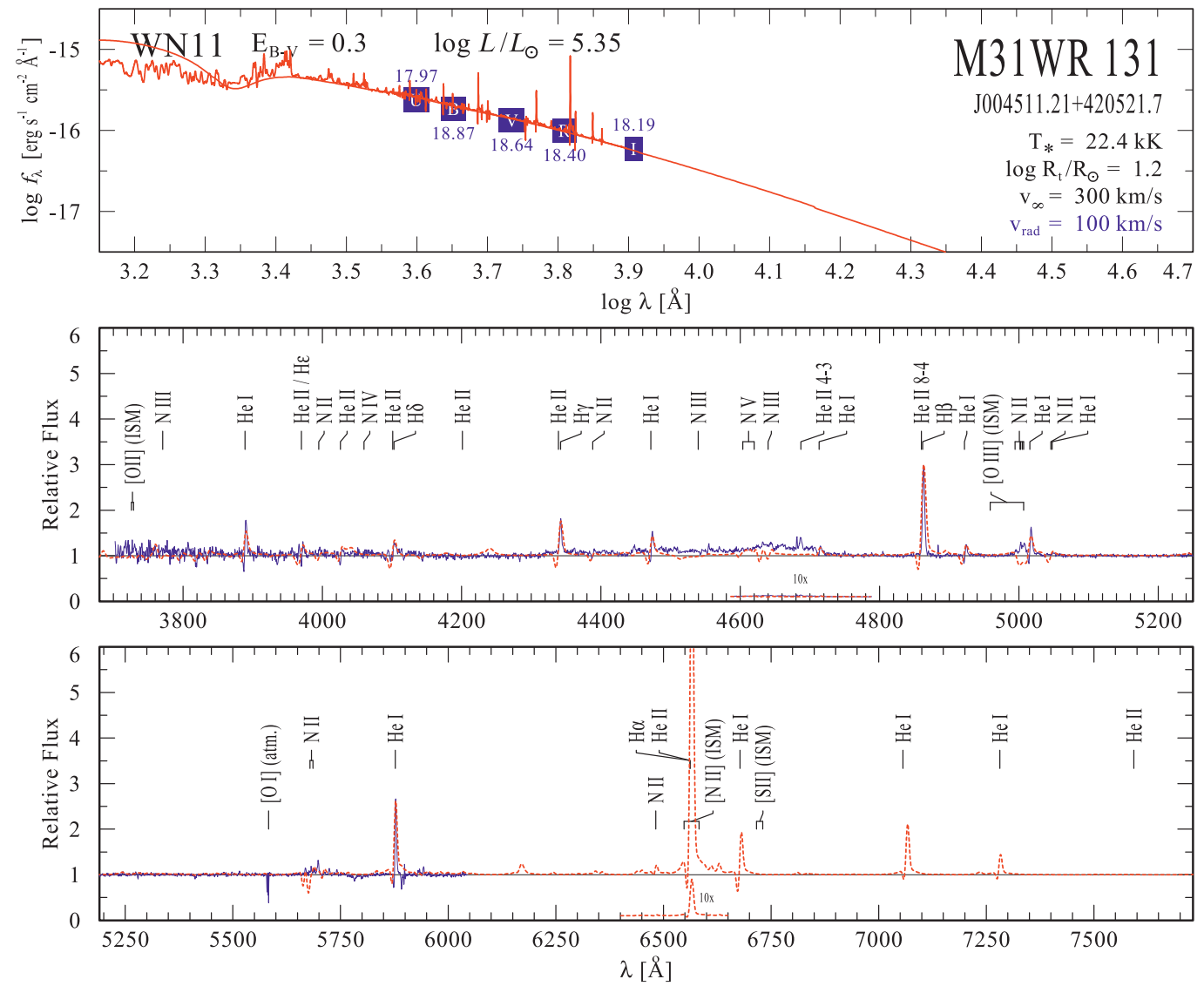

Fig. A.16. Spectral fit for M31WR 131. 
A. Sander et al.: The Wolf-Rayet stars in M31. I.
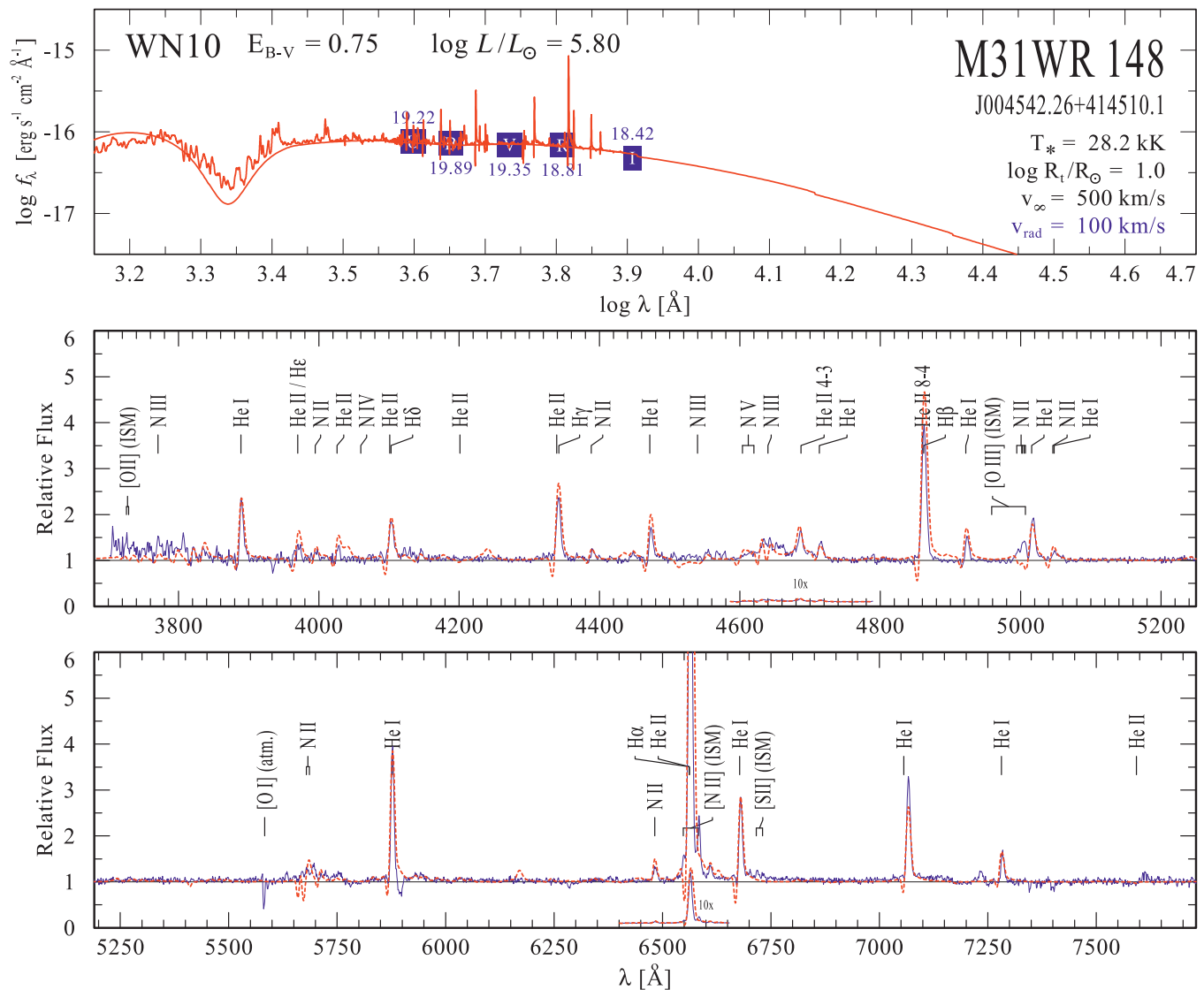

Fig. A.17. Spectral fit for M31WR 148. 


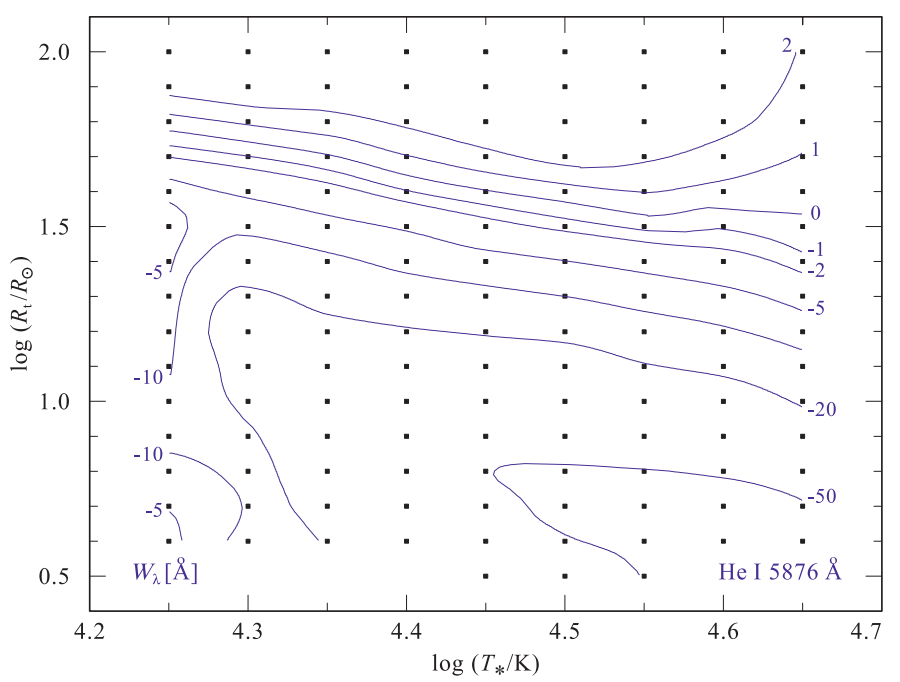

Fig. B.1. Contours of constant equivalent width $W_{\lambda}$ for He I $5876 \AA$ in the WNh model grid with $X_{\mathrm{H}}=0.2$ and $v_{\infty}=500 \mathrm{~km} \mathrm{~s}^{-1}$. A positive value indicates an absorption line, negative values refer to emission lines.

\section{Appendix B: Problematic objects}

\section{B.1. M31WR 60}

For the WN10 star \#60 (J004242.33+413922.7), we could not reproduce the peak heights of the unusually strong He I lines. Looking into the model grids reveals that there seems to be a maximum for the He r emission lines that cannot be superseded for a given temperature (and chemical composition), even if we further increase the mass-loss rate. This is illustrated in Fig. B.1 where we plot contours of constant equivalent width $W_{\lambda}$ for one of our WNh grids. The temperature $T_{*}$ is fixed by He II $4686 \AA$ and $\mathrm{N}_{\text {III }} 4634 / 42 \AA$, which are reproduced well in our fit (cf. Fig. A.8).

For \#60, we therefore focused on the smaller emission lines that are mostly reproduced. The best result is obtained for a model with $20 \%$ hydrogen, even though the $\mathrm{H} \beta, \mathrm{H} \gamma$, and $\mathrm{H} \delta$ lines are slightly too weak in the model. However, models with $35 \%$ hydrogen or more do not lead to a better fit of the whole spectrum.

\section{B.2. $M 31 W R 42$}

Another problematic object is \#42, alias J004130.37+410500.9. This spectrum is highly contaminated with strong nebular emission. Massey et al. (2007) refrained from assigning a subtypes and listed the star simply as WNL. Neugent et al. (2012) speculated that it might be of WN7 subtype, but have not included this in their table either. Unfortunately the observed spectrum ends at $6000 \AA$ so that $\mathrm{H} \alpha$ is not covered. Only two prominent WN features are available for the diagnostic, $\mathrm{N}_{\text {III }} 4634 / 42 \AA$ and He II $4686 \AA$, both in emission. Indeed the roughly similar peak heights of these two lines would classify the star as WN7 following the scheme of van der Hucht (2001), but since N Iv is so weak in the spectrum, it could also be classified as WN8. However, we do not see strong P-Cyg-profiles that would be another criterion for the WN8 subtype. We therefore specify the subtype as WN78 until future observations become available.

From the two diagnostic lines we can obtain the basic parameters $T_{*}$ and $R_{\mathrm{t}}$ for $\# 42$. Owing to the strong nebular emission lines, the hydrogen content $X_{\mathrm{H}}$ and the terminal velocity $v_{\infty}$ are harder to determine. From the unblended lines, neither the $\mathrm{N}$ IIIcomplex at 4634-42 $\AA$ nor the weak He II $4686 \AA$ line are really sensitive to $v_{\infty}$. We therefore use the $\mathrm{HI}$ and $\mathrm{He}_{\mathrm{I}}$ lines, although they are polluted with nebular emission. We can rule out velocities higher than $v_{\infty}=500 \mathrm{~km} \mathrm{~s}^{-1}$ because they would produce line profiles that are broader than the observed lines. The best compromise is obtained with $v_{\infty}=300 \mathrm{~km} \mathrm{~s}^{-1}$, which would favor WN8 over the WN7 subtype classification, where we usually find higher terminal velocities.

For the hydrogen content, we use the indirect sensitivity of He II $4686 \AA$ to rule out models with $50 \%$ hydrogen and zero hydrogen. We favor a model with $20 \%$ over those with $35 \%$ hydrogen because we observe a small emission of $\mathrm{C}_{\text {III }} 4650 \AA$ and therefore expect a more "evolved" star, but a larger uncertainty than for the other objects remains. 